\title{
Wie ungleich ist die Welt? Eine empirische Analyse
}

\author{
Norbert Berthold* \\ Bayerische Julius-Maximilians- Universität Würzburg
}

\author{
Alexander Brunner \\ Bayerische Julius-Maximilians-Universität Würzburg
}

\section{Einleitende Bemerkungen}

Die Entwicklung der weltweiten Ungleichheit ist ein kontrovers diskutiertes Thema. In Presse und Öffentlichkeit erhitzen sich die Gemüter an der Frage, weshalb manche Länder der Welt reich, andere hingegen arm sind. Die im letzten Jahr aufgekommene Occupy Bewegung entstand auch aus der empfundenen Ungerechtigkeit darüber, dass Teile der Menschheit mit weniger als einem Dollar pro Tag ihren Lebensunterhalt bestreiten müssen, während andere Menschen Milliardäre sind. Bevor eine Antwort auf diese Probleme überhaupt gefunden werden kann, muss jedoch zunächst geklärt werden, wie ungleich die Welt eigentlich ist und wie sich diese Ungleichheit im Laufe der Zeit verändert hat. Ziel dieses Papiers ist es daher, einen Beitrag zu der aktuellen Diskussion und eine Übersicht über die tatsächliche Höhe sowie die Entwicklung der Ungleichheit der Welt zu liefern. Dabei reiht sich der Beitrag in eine Reihe von Publikationen der Weltbank (Milanovic 2005, Weltbank 2006, Milanovic 2007, 2009), die sich intensiv mit dem vorliegenden Problem befasst haben, ein.

Gegenstand des vorliegenden Papiers ist es, die momentane Entwicklung in der Literatur aufzugreifen und diese um eigene Berechnungen zu ergänzen. Dabei liegt ein Schwerpunkt auf dem Vergleich der Ergebnisse anhand unterschiedlicher Datensätze mit den Berechnungen anderer Autoren. Dieses Vorgehen hat zum Ziel, Gemeinsamkeiten und Unterschiede aufzudecken, die sich anhand der unterschiedlichen Datenquellen ergeben, sowie Rückschlüsse auf die bestehenden Datenprobleme zu ziehen. Während für eine Analyse der weltweiten Ungleichheit üblicherweise auf einen Datensatz zurückgegriffen wird, wird in dieser Abhandlung explizit derselbe Untersuchungsgegenstand anhand von verschiedenen Datenquellen betrachtet. Während bspw. Milanovic (2009) in seiner Studie alleine die Daten der Weltbank für seine Berechnungen der weltweiten Ungleichheit heranzieht, werden in diesem Beitrag auch die Daten der Penn World Tabelles, der Total Economy Database und die Angus Maddison Daten genutzt. Wie sich im Laufe der Arbeit herausstellen wird, unterscheiden sich dabei die Ergebnisse teilweise so diametral, dass eine eindeutige Bestandsaufnahme über die Entwicklung der Ungleichheit in den letzten Jahrzehnten

\footnotetext{
*Korrespondenzadresse: Lehrstuhl für Volkswirtschaftslehre, insbes. Wirtschaftsordnung und Sozialpolitik, Bayerische Julius-Maximilians-Universität Würzburg, Sanderring 2, D-97070 Würzburg, Tel.: +49931-3182925, Fax: +49931-3182774, E-Mail: norbert.berthold@uni-wuerzburg.de. - Die Autoren bedanken sich bei Lars P. Feld und einem anonymen Gutachter für wertvolle Hinweise.
} 
nicht- oder nur eingeschränkt - erfolgen kann. Dieses Ergebnis hat auch eine hohe politische Relevanz, da es mithin nur bedingt möglich ist, entwicklungspolitische Maßnahmen zu beurteilen, wenn der Erfolg oder Misserfolg über einen langen Zeitraum nicht $\mathrm{zu}$ bestimmen ist. Somit trägt dieser Beitrag zur aktuellen Diskussion um die Entwicklung der weltweiten Ungleichheit bei, da viele der vorschnell aufgestellten Behauptungen nicht eindeutig zu belegen sind.

Im weiteren Verlauf der Untersuchung wird zunächst in Kapitel 2 die zugrunde liegende Problematik und der Begriff der Ungleichheit aus einer globalen Perspektive erläutert. Kapitel 3 widmet sich einer kurzen Beschreibung der Daten, bevor in Kapitel 4 eigene Ergebnisse präsentiert und mit anderen Untersuchungen $\mathrm{zu}$ diesem Themenbereich verglichen werden. Hier erfolgt eine Interpretation der Ergebnisse und eine Diskussion über deren Aussagekraft, bevor der Beitrag mit einer kurzen Zusammenfassung in Kapitel 5 schließt.

\section{Weltweite Ungleichheit}

Methodisch unterscheidet sich die Messung der weltweiten Ungleichheit nicht von der Berechnung der personellen Ungleichheit. Somit kann auf die üblichen Ungleichheitsmaße aus der einschlägigen Literatur (Sen 1973, Atkinson 1983, Cowell 2000, Jenkins 1991, sowie Jenkins und van Kerm 2009) zurückgegriffen werden. Jedoch kann der Begriff der weltweiten Ungleichheit unterschiedlich definiert werden. Entscheidend ist hier die Frage: Ungleichheit von wem? Dazu wurden drei verschiedene Konzepte entwickelt (Weltbank 2006, Milanovic 2007). Das erste Konzept, die sogenannte (ungewichtete) internationale Ungleichheit (Konzept 1), bezieht sich auf die Ungleichheit zwischen Nationen. Entsprechend kann ein Ungleichheitsmaß wie bspw. der Gini-Koeffizient auf Basis der BIP pro Kopf Daten der Länder berechnet werden. Der Nachteil dieses Vorgehens ist, dass Einkommensunterschiede innerhalb des Landes vernachlässigt werden und einem Land wie Luxemburg das gleiche Gewicht zukommt wie China. Die letztgenannte Überlegung führt zum zweiten Konzept, der gewichteten internationalen Ungleichheit (Konzept 2). Im Unterschied zur ersten Herangehensweise wird dabei das BIP pro Kopf mit der Anzahl der Einwohner gewichtet. Dies führt dazu, dass bevölkerungsreiche Länder einen deutlich höheren Einfluss auf ein Ungleichheitsmaß haben. Das ist grundsätzlich eine wünschenswerte Eigenschaft, da damit berücksichtigt wird, wie viele Personen in einem Land mit einem gegebenen Wohlstandsniveau leben. Der Nachteil dieses Vorgehens ist, dass weiterhin implizit eine völlige Gleichverteilung des Einkommens innerhalb des Landes unterstellt wird. Dieser Mangel wird schließlich mit dem dritten Konzept, der globalen Ungleichheit, behoben (Konzept 3). Hier werden Haushaltsdaten aller Länder zusammengefügt, so dass tatsächlich die Ungleichheit auf individueller Ebene berechnet wird. Eine Übersicht dieser drei Konzepte ist Tabelle $1 \mathrm{zu}$ entnehmen.

Entsprechend ist das dritte Konzept das geeignetste Vorgehen, um eine Aussage über die Ungleichheit in der Weltbevölkerung treffen zu können. Jedoch ist diese Herangehensweise auch mit den größten Problemen behaftet. Im Idealfall müsste eine einzige Umfrage durchgeführt werden, um eine repräsentative Stichprobe der Weltbevölkerung $\mathrm{zu}$ erhalten. Dies ist faktisch unmöglich. Die nächstliegende Möglichkeit besteht darin, die auf nationaler Ebene erhobenen Datensätze zusammenzufügen, was sich als praktikable 
Tabelle 1 Konzepte der Ungleichheitsmessung

\begin{tabular}{|c|c|c|c|}
\hline & Konzept 1 & Konzept 2 & Konzept 3 \\
\hline & $\begin{array}{l}\text { Ungewichtete } \\
\text { internationale } \\
\text { Ungleichheit }\end{array}$ & $\begin{array}{l}\text { Gewichtete } \\
\text { internationale } \\
\text { Ungleichheit }\end{array}$ & $\begin{array}{l}\text { Globale } \\
\text { Ungleichheit }\end{array}$ \\
\hline Datenquelle & VGR & VGR & Haushaltsdaten \\
\hline Beobachtungseinheit & Land & $\begin{array}{c}\text { Land (gewichtet mit } \\
\text { der Bevölkerung) }\end{array}$ & Individuum \\
\hline Wohlfahrtsmaß & $\begin{array}{l}\text { BIP pro Kopf (oder } \\
\text { andere VGR } \\
\text { Größen) }\end{array}$ & $\begin{array}{l}\text { BIP pro Kopf (oder } \\
\text { andere VGR } \\
\text { Größen) }\end{array}$ & $\begin{array}{l}\text { Verfügbares } \\
\text { Einkommen oder } \\
\text { Ausgaben }\end{array}$ \\
\hline $\begin{array}{l}\text { Währungsumrechnung } \\
\text { Ungleichheit innerhalb } \\
\text { des Landes }\end{array}$ & Ignoriert & $\begin{array}{l}\text { Wechselkurse oder } \\
\text { Ignoriert }\end{array}$ & $\begin{array}{l}\text { Kaufkraftstandards } \\
\text { Berücksichtigt }\end{array}$ \\
\hline
\end{tabular}

Quelle: Eigene Darstellung in Anlehnung an Milanovic (2007), S. 28.

Lösung anbietet. Damit entsteht jedoch eine Reihe weiterer Probleme, welche beachtet werden müssen. An erster Stelle steht dabei die Vergleichbarkeit der Datensätze (vgl. Atkinson und Brandolini, 2001). Inkonsistenzen können insbesondere bei der Auswahl des Untersuchungsgegenstandes (Einkommen versus Konsum), der Untersuchungseinheit (Haushaltsdefinition), der Datenquelle (staatlich erhobene Daten versus freiwillige Umfragen), der Einkommensdefinition und der Berücksichtigung nicht-monetärer Einkommensbestandteile entstehen. ${ }^{1}$ Es gibt zwar einige Projekte, die sich mit der Standardisierung von unterschiedlichen Datenquellen befassen (bspw. die Luxembourg Income Study (LIS)), jedoch können auch dort nicht alle Probleme gelöst werden (Atkinson, Rainwater und Smeeding 1995, S. 25-38). Darüber hinaus liegen dann meist nur Daten für eine geringe Anzahl an Ländern und Zeitpunkten vor, was die Nutzbarkeit deutlich einschränkt. Insgesamt sind Versuche, eine einzige weltweite Einkommensverteilung $\mathrm{zu}$ berechnen, mit großer Vorsicht zu genießen, da bereits der Vergleich verschiedener Maßzahlen zwischen Ländern deutliche Probleme aufweist (Atkinson und Brandolini 2001).

Neben der Frage der Ungleichheit „von wem“, ist bei den drei Konzepten insbesondere auch die Ungleichheit „von was" zu eruieren. Bei der globalen Ungleichheit kann wiederum auf die Literatur zur personellen Ungleichheit zurückgegriffen werden (UN 1977, Atkinson, Rainwater und Smeeding 1995). ${ }^{2}$ Bei der gewichteten und ungewichteten internationalen Ungleichheit hingegen muss eine entsprechende Pro-Kopf-Größe herangezogen werden. Das am weitesten verbreitete Konzept stellt dabei das BIP pro Kopf dar. Anstatt dieser Größe (Milanovic 2009) kann auch das BSP/BNEpro Kopf (Milanovic 2007) und somit das Inländerkonzept herangezogen werden. Andere denkbare Definitionen sind das Nettonationaleinkommen, also das BSP abzüglich der Abschreibungen, und das

1. Insbesondere sind hier kalkulatorische Mieten für selbstgenutzten Wohnraum gemeint.

2. Um diese Frage zu beantworten, ist insbesondere die Wahl der Untersuchungseinheit, also Einkommen oder Konsum und die jeweilige Abgrenzung relevant. Des Weiteren fällt in diesen Bereich die Wahl der Äquivalenzskalen. 
Volkseinkommen. Bei letzterem wird noch der Saldo aus Abgaben an den Staat und erhaltene Subventionen abgezogen. ${ }^{3}$ Bei diesen Definitionen nähert man sich dabei immer weiter dem aus der Literatur der personellen Einkommensverteilung bekannten Begriff des verfügbaren Einkommens an. Jedoch ist vor allem auf der Makroebene und beim Ländervergleich die Bereitstellung öffentlicher Güter ein wesentlicher Faktor. Wird das Volkseinkommen pro Kopf berechnet, wird dieser Teil des Wohlstandes nicht berücksichtigt. Ein weiterer Nachteil der letztgenannten Definitionen besteht darin, dass vergleichbare internationale Daten deutlich seltener vorhanden sind als beim BIP pro Kopf.

Grundsätzlich ist für die Untersuchung der Ungleichheit die Art der verwendeten Größe genau dann nicht entscheidend, wenn die Relation zweier Größen (bspw. Nettonationaleinkommen geteilt durch Bruttoinlandsprodukt) über die Zeit und zwischen den Ländern konstant ist. In einem solchen Fall hat die Wahl der Größe keinen Einfluss auf die Ungleichheitsmaße. Diese Eigenschaft, welche die hier genutzten Ungleichheitsmaße besitzen, wird als Skaleninvarianz bezeichnet. Entsprechend ist es dann irrelevant, wenn man eine andere Größe als das Volkseinkommen pro Kopf heranzieht. Insgesamt überwiegt somit der Vorteil der breiten Verfügbarkeit des BIP pro Kopf. Die definitorischen Nachteile werden durch die höhere Anzahl unterschiedlicher Datensätze überkompensiert.

Allen drei Konzepten ist unabhängig von der Einkommensdefinition das Problem der Währungsumrechnung gemein. Prinzipiell bietet es sich an, entweder auf Wechselkurse oder Kaufkraftstandards zurückzugreifen. Erstere haben den Nachteil, dass nicht-handelbare Waren und Dienstleistungen im Kurs nicht einbezogen werden. Kaufkraftstandards berücksichtigen hingegen alle handelbaren und nicht-handelbaren Güter. Sie weisen jedoch das Problem auf, dass sie nicht am Markt beobachtbar sind, sondern berechnet werden müssen. Um einen Eindruck über die tatsächliche weltweite Ungleichheit und Armut zu erhalten, ist die Berechnung der Kaufkraftstandards jedoch unerlässlich (Deaton 2010, S. 2-5). Vor diesem Hintergrund beschäftigt sich beispielsweise die Weltbank im Rahmen des International Comparison Program (ICP) intensiv mit der Berechnung geeigneter Umrechnungspreise. Die Ergebnisse der letzten Runde (ICP 2005), welche im Jahr 2008 vorgestellt wurden, führten zu einer massiven Korrektur der Umrechnungskurse (Milanovic 2009, S. 2). So wurde beispielsweise das BIP pro Kopf in KKS für China und Indien deutlich nach unten korrigiert $(-38,7 \%$ bzw. $-37,2 \%)$, während es in Mexiko und Nigeria erheblich stieg (+10,0\% bzw. $+26,7 \%)$ (Milanovic 2009, S. 6). Es ist offensichtlich, dass solch massive Verwerfungen einen erheblichen Einfluss auf die gemessene Ungleichheit und Armut in der Welt haben. Vor diesem Hintergrund ist es durchaus zu erwarten, dass Berechnungen auf Grundlage unterschiedlicher Daten zu abweichenden Ergebnissen führen.

\section{Datenlage}

Zur Messung der gewichteten und ungewichteten internationalen Ungleichheit ist es notwendig, BIP pro Kopf Daten in Kaufkraftstandards zu verwenden. Tatsächlich existiert hierzu eine Reihe von Datenquellen. Der Vorteil dieser Vielfalt

3. Vgl. zu den jeweiligen Berechnungen die ausführliche Darstellung in Brümmerhoff (2007), S. 54-75. Eine Übersicht ist dabei auf S. 55 zu finden. 
Tabelle 2 Datenquellen

\begin{tabular}{|c|c|c|c|}
\hline Anbieter & Datenbank & Version & Quelle \\
\hline Weltbank & $\begin{array}{l}\text { World } \\
\text { Development } \\
\text { Indicators (WDI) }\end{array}$ & April 2010 & $\begin{array}{l}\text { http://data.worldbank.org/data- } \\
\text { catalog/world-development- } \\
\text { indicators }\end{array}$ \\
\hline $\begin{array}{r}\text { Universität von } \\
\text { Pennsylvania }\end{array}$ & $\begin{array}{l}\text { Penn World Tables } \\
\text { (PWT) }\end{array}$ & PWT 6.3 & http://pwt.econ.upenn.edu/ \\
\hline $\begin{array}{l}\text { Universität } \\
\text { Groningen }\end{array}$ & $\begin{array}{l}\text { Total Economy } \\
\text { Database (TED) }\end{array}$ & $\begin{array}{l}\text { Januar } \\
2010\end{array}$ & $\begin{array}{l}\text { http://www.ggdc.net/databases/ } \\
\text { ted.htm }\end{array}$ \\
\hline Maddison & $\begin{array}{l}\text { Maddison Series } \\
\text { (Maddison) }\end{array}$ & $2-2010$ & http://www.ggdc.net/maddison/ \\
\hline
\end{tabular}

liegt darin, dass die Berechnung der Ungleichheit auf unterschiedlichen Quellen beruht und somit nicht auf einen einzelnen Datenproduzenten vertraut werden muss. Entsprechend können Gemeinsamkeiten und Unterschiede herausgearbeitet werden. Es besteht dann aber der Nachteil, dass sich die Ergebnisse gegebenenfalls widersprechen und somit keine endgültigen Schlüsse gezogen werden können.

Um den Einfluss der Wahl einer bestimmten Datenquelle abschätzen zu können, soll daher in den nachfolgenden Untersuchungen auf verschiedene Datensätze zurückgegriffen werden. Dies bietet die Möglichkeit, die Ergebnisse miteinander zu vergleichen und entsprechend zu eruieren, ob sich ein konsistentes Bild abzeichnet. Die Grundlage der nachfolgenden Berechnungen bilden dabei die Bevölkerungsund BIP pro Kopf Daten der Weltbank (WDI), der Universität von Pennsylvania (PWT), der Universität Groningen (TED) und Angus Maddison (Maddison). Ein entsprechender Überblick ist in der nachfolgenden Tabelle $2 \mathrm{zu}$ finden. Dabei ist zu beachten, dass die TED zwei unterschiedliche BIP pro Kopf Zeitreihen mit unterschiedlichen Kaufkraftparitäten bereitstellt. Diese werden nachfolgend als TED GK (Geary Khamis) und TED EKS bezeichnet.

Darüber hinaus ist es an dieser Stellte wichtig darauf hinzuweisen, dass die Anzahl der berücksichtigten Länder zwischen und innerhalb der Datensätze schwankt. Dies lässt sich beispielsweise mit den fehlenden Daten der ehemaligen Mitgliedsstaaten des Warschauer Pakts begründen. Auch für einige Entwicklungsländer liegen verlässliche Daten selten für einen langen Zeitraum vor. Da tendenziell davon auszugehen ist, dass Daten für Entwicklungs- und Schwellenländer erst seit wenigen Jahren zur Verfügung stehen, ist mit einem erheblichen Selektionsbias zu rechnen. Dabei ist es fraglich, ob diese Effekte einen einmaligen Niveaueffekt darstellen oder die gesamte Entwicklung verzerrt ist. Es ist offensichtlich, dass der Eintritt neuer Länder in den Datensatz insbesondere einen großen Einfluss auf die ungewichtete internationale Ungleichheit hat, da hier jedes Land mit dem Gewicht "1" eingeht, das heißt bei einhundert Ländern im Datensatz kann die neue Verfügbarkeit von zehn weiteren Ländern ab einem gewissen Zeitpunkt einen sehr starken Einfluss haben. Bei den anderen beiden Konzepten der Ungleichheit kommt es hingegen auf die Relation der Bevölkerung der neu hinzu gekommenen Länder und der bereits im Datensatz vorhandenen Länder an, ob und wie stark ein Ungleichheitsmaß durch den Eintritt neuer Länder in den Datensatz beeinflusst wird. 


\section{Ergebnisse}

\subsection{Entwicklung der ungewichteten internationalen Ungleichheit}

In diesem Kapitel soll die Entwicklung der weltweiten Ungleichheit anhand verschiedener Datensätze und den drei in Kapitel 2 vorgestellten Konzepten diskutiert werden. Zunächst wird anhand des Theil-Indexes und des GiniKoeffizienten der Verlauf der ungewichteten internationalen Ungleichheit(Konzept 1) beleuchtet. ${ }^{4}$ Deren Entwicklung kann Abbildung 1 entnommen werden. Dabei ist festzuhalten, dass sich die berechneten Ungleichheitsmaße zwischen den verschiedenen Datensätzen in ihrer absoluten Höhe deutlich unterscheiden. So lag der Gini-Koeffizient bei der TED (EKS) Zeitreihe in den 60er Jahren bei etwa 65 Gini-Punkten, während der Wert bei den Daten der PWT deutlich unter 50 lag. Betrachtet man jedoch die Trends, so sind mehrere Übereinstimmungen zu erkennen. So hat die ungewichtete internationale Ungleichheit (eine Ausnahme bilden die Ergebnisse der PWT) in den sechziger Jahren leicht abgenommen. Anfang der 70er Jahre ist eine leichte Zunahme der Ungleichheit zu beobachten, während ab Mitte des Jahrzehnts bis zur Mitte des Folgejahrzehnts die Verteilung des BIP pro Kopf wieder etwas gleicher wurde. Zu Anfang der 90er Jahre ist wiederum ein schwacher Anstieg zu beobachten. Ab Mitte der 90er Jahre nahmen sowohl GiniKoeffizient als auch Theil-Index wieder zu. Um die Jahrhundertwende kam es zu einer Trendumkehr. Die Ungleichheit fiel bei allen Datensätzen deutlich. Nur bei den PWT ist ab der Mitte des Jahrzehnts ein leichter Anstieg zu erkennen. Die beschriebenen Entwicklungen sind im Wesentlichen für beide Ungleichheitsmaße identisch.

Besonders auffällig am Verlauf der Zeitreihen in Abbildung 1 sind der rapide Anstieg der Ungleichheitsmaße bei den WDI am Anfang der 60er Jahre sowie die in fast allen Datensätzen beobachtbaren Zacken zu Beginn der 70er Jahre und Ende der 80er Jahre. Zu diesen Zeitpunkten kamen jeweils neue Länder hinzu (vgl. Abbildung 2). Bei den Maddison und TED (GK) Daten wurden beispielsweise zu Beginn der 70er Jahre einmalig Daten für einzelne Entwicklungsländer ausgewiesen, die dann erst sehr viel später wieder berücksichtigt wurden. Es sticht zudem heraus, dass nach Ende der 80er Jahre ein Bruch in allen Reihen vorzuliegen scheint. Dies ist durch die Hinzunahme der ehemaligen Mitglieder des Warschauer Paktes zu erklären. Anschließend ist der Verlauf der Zeitreihen folgerichtig ähnlicher, da sich die Anzahl der Beobachtungen nur geringfügig ändert. Entsprechend können die vorherigen Ergebnisse durchaus ein statistisches Artefakt sein, da im Zeitablauf immer wieder für neue Länder Daten verfügbar werden. Geht man davon aus, dass jene arme Nationen sind, so bedeutet dies, dass durch deren Zutritt die gemessene Ungleichheit ansteigt. Dieser Effekt ist erst ab Ende der 80er Jahre nicht mehr gegeben, weil ab dann für fast alle Länder Daten vorliegen. Somit kann eine konsistente Aussage über die internationale Ungleichheit streng genommen erst ab Anfang der 90er Jahre gemacht werden. Die Entwicklung der in den Datensätzen berücksichtigten Länder ist Abbildung 2 zu entnehmen. Hier ist für die meisten Datensätze ein Sprung um das Jahr 1970 und 1990 zu erkennen,

4. Zur Definition dieser Ungleichheitsmaße vgl. Sen (1973), Atkinson (1983), Cowell (2000), Jenkins (1991) sowie Jenkins und van Kerm (2009). Sowohl der Gini-Koeffizient als auch der Theil-Index sind Ungleichheitsmaße, d.h., dass ein höherer Wert jeweils eine höhere Ungleichheit impliziert. 


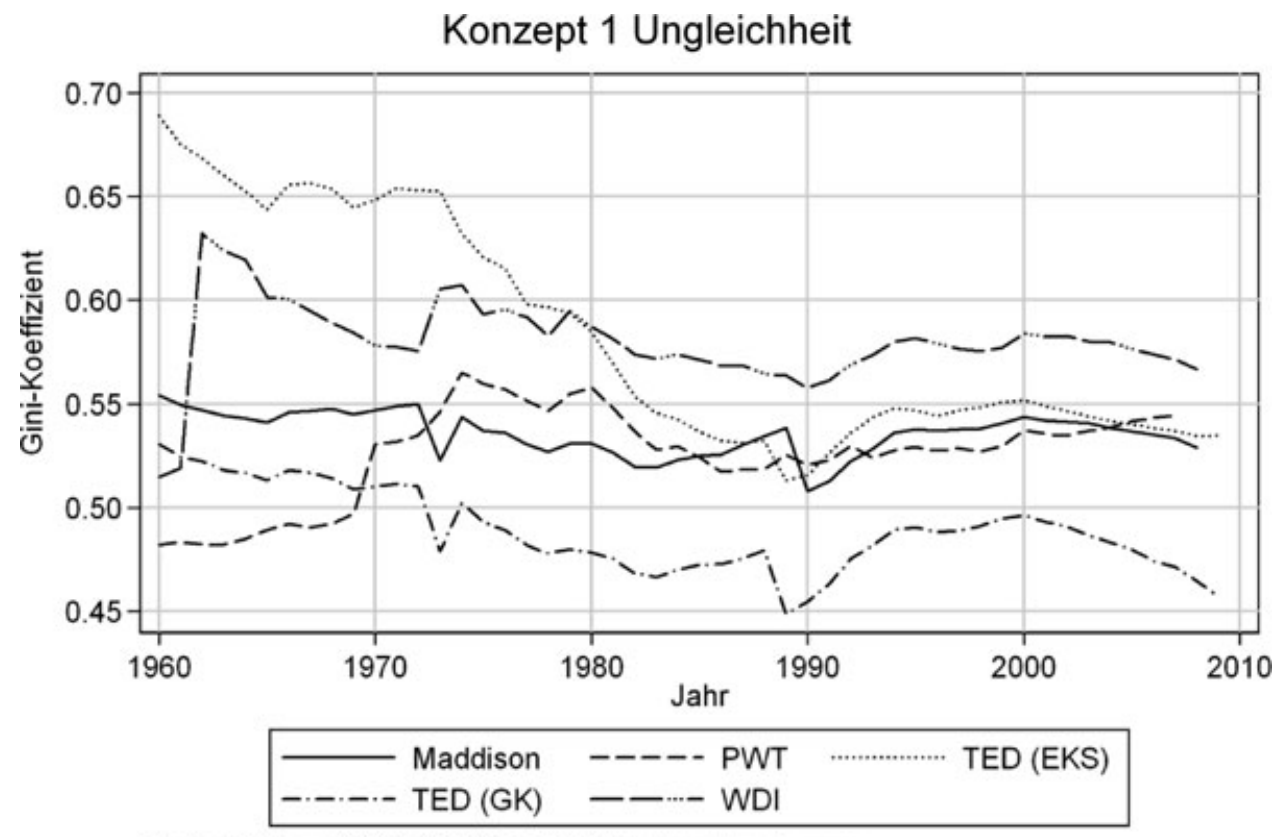

Quelle: Maddison (2010), PWT 6.3, TED, WDI; eigene Berechnungen.

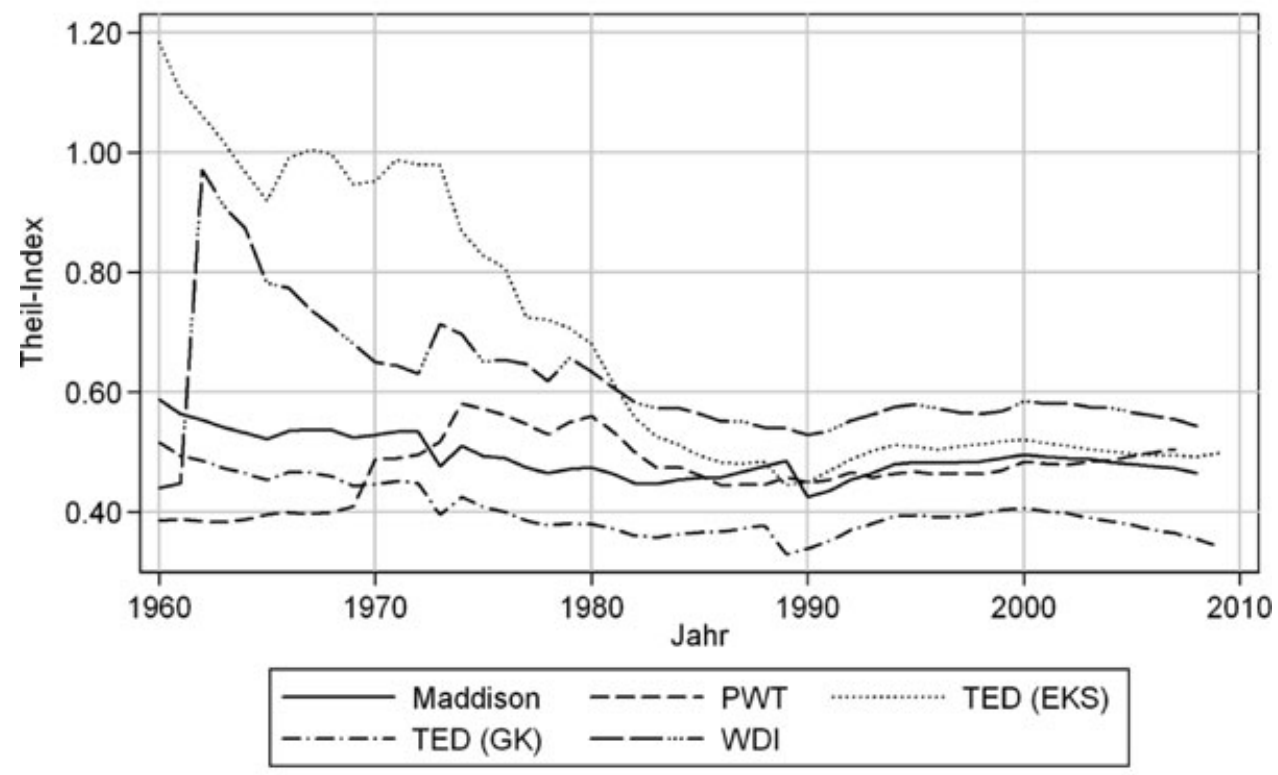

Quelle: Maddison (2010), PWT 6.3, TED, WDI; eigene Berechnungen.

Abbildung 1 Entwicklung der ungewichteten internationalen Ungleichheit

was sich auch in den berechneten Ungleichheitsmaßen widerspiegelt. Dabei fällt auf, dass sich insbesondere in den Weltbankdaten die Anzahl der Beobachtungen ständig ändert und im Zeitablauf kontinuierlich zunimmt. Dieser Effekt kann das beobachtbare Bild verzerren, da selbst die Hinzunahme eines einzelnen Landes starke Auswirkungen auf die Ungleichheit haben kann. So ist in Abbildung 1 der 
Wie ungleich ist die Welt? Eine empirische Analyse

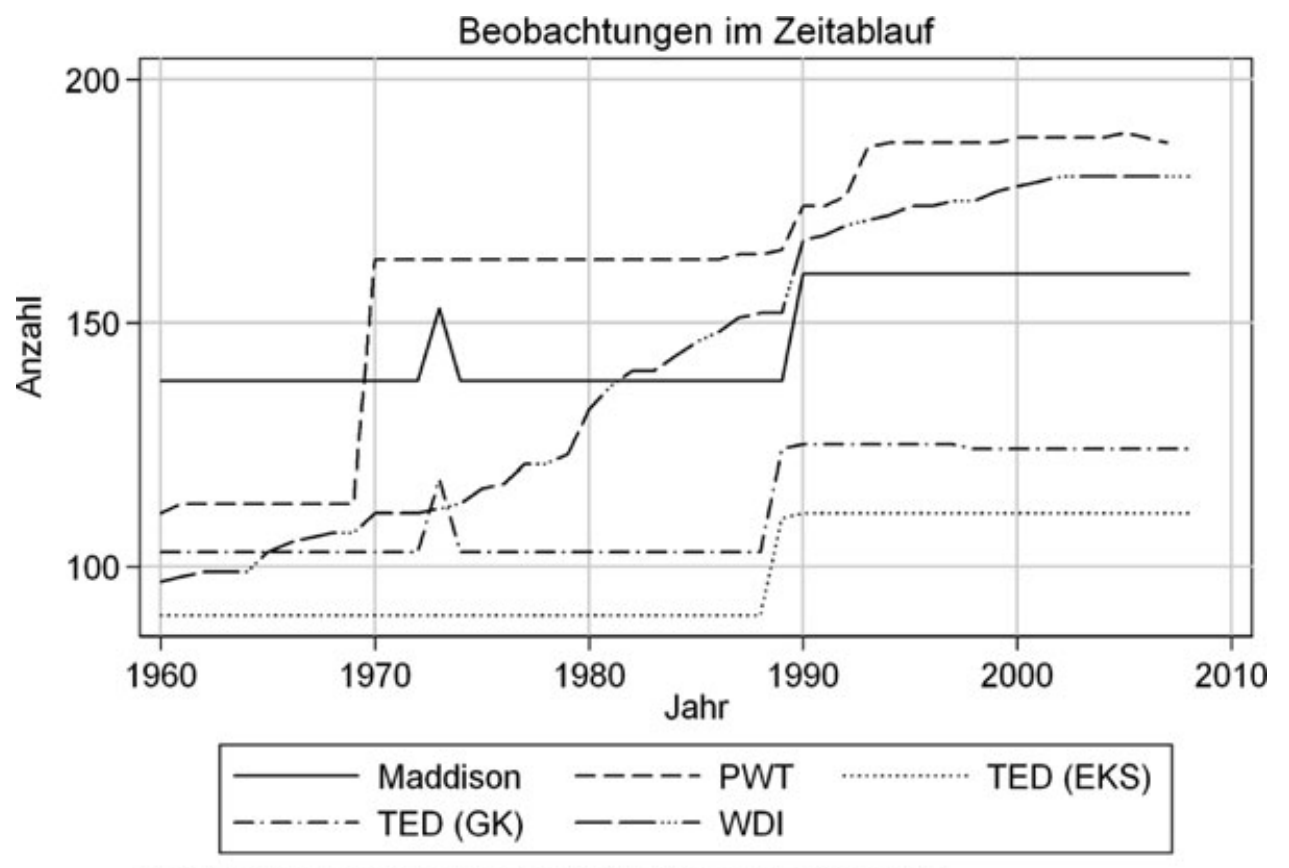

Quelle: Maddison (2010), PWT 6.3, TED, WDI; eigene Berechnungen.

Abbildung 2 Anzahl der Beobachtungen im Zeitablauf

sprunghafte Anstieg des Gini-Koeffizienten in der WDI-Zeitreihe auffällig. Dieser steigt zwischen 1961 und 1962 von 0,52 auf 0,63. Die Anzahl der Beobachtungen hat sich jedoch in diesem Zeitraum kaum geändert (vgl. Abbildung 2). In der Tat kam nur ein einziges Land, nämlich Kuwait, in den Datensatz hinzu. Jedoch lag 1962 in diesem Land das BIP pro Kopf gemessen in 2005 KKS bei 155568 \$ und war damit mehr als sechseinhalb (sic!) mal so hoch wie der Wert des zweitreichsten Landes. ${ }^{5}$ Berechnet man den Gini für das Jahr 1962 für alle Länder außer Kuwait, so liegt er weiterhin bei 0,52. Spätestens an dieser Stelle wird klar, dass die Verzerrungen durch die sich ändernde Anzahl an Beobachtungen alles andere als vernachlässigbar sind. Dieses Problem kann nicht befriedigend gelöst werden. Wenn man nur Länder berücksichtigt, für welche zu jedem Zeitpunkt Daten vorliegen, schließt man einen großen Teil der Daten aus. Da es sich dabei vornehmlich um Entwicklungs- und Schwellenländer handelt, würde sich somit die Messung der Ungleichheit auf die relativ wohlhabenden Länder konzentrieren und dem Anspruch, die Ungleichheit auf globaler Ebene zu messen, zuwiderlaufen.

Die Frage, ob die Ungleichheit zum Ende des Beobachtungszeitraums geringer ist als am Anfang, kann nicht eindeutig beantwortet werden. So nahmen bei der Maddison-Zeitreihe sowohl Gini $(0,55$ auf 0,53$)$ als auch Theil $(0,59$ auf 0,46$)$ zwischen 1960 und 2008 ab, während bei den PWT beide Maße zwischen 1960 und 2007 (0,48 auf 0,54 bzw. 0,39 auf 0,50) zunahmen. Die beiden TED-Reihen wiesen eine Abnahme der Ungleichheit auf, während bei den WDI eine Zunahme zu beobachten war. Somit scheint die Aussagekraft zunächst gering. Eine Erklärung 
für diese unterschiedlichen Ergebnisse liegt jedoch darin begründet, dass sich die Anzahl der Beobachtungen sowohl innerhalb der Datensätze als auch zwischen den Datensätzen deutlich unterscheiden (vgl. Abbildung 1). Beachtet man jedoch, dass bei den Daten der PWT ein offensichtlicher Strukturbruch vorliegt ${ }^{6}$ und dass bei den WDI-Daten der Kuwait-Effekt vorliegt, so ist es sinnvoller, die Werte Mitte der 70er Jahre mit dem Ende des Beobachtungszeitraums zu vergleichen. Hier weisen dann alle Zeitreihen auf eine Abnahme der Ungleichheit im Beobachtungszeitraum hin. Letztlich besteht eine andere Möglichkeit darin, eben keine Aussage über den gesamten Zeitraum, sondern lediglich Aussagen über die einzelnen Zeitabschnitte zu treffen, in denen die Anzahl der Länder in den Datensätzen relativ stabil ist.

Um eine Vorstellung vom Verlauf der Ungleichheit über einen längeren Zeitraum zu gewinnen, kann zusätzlich ein Panel mit einer fixen Anzahl an Beobachtungen konstruiert werden. Dieses Vorgehen ermöglicht es zumindest, eine konsistente Aussage über die Entwicklung der ungewichteten internationalen Ungleichheit dieser Länder zu treffen. Eine entsprechende grafische Darstellung ist in Abbildung 11 auf S. 22 im Anhang zu finden. Dabei wurden alle Länder berücksichtigt, für die seit dem Jahr 1970 durchgehend Beobachtungen vorlagen. Die Ergebnisse unterscheiden sich zwischen den verschiedenen Datensätzen zunächst recht deutlich. Ab Mitte der 80er nimmt die Ungleichheit jedoch für alle Datenquellen $\mathrm{zu}$ und nach der Jahrtausendwende deutet sich bei der Mehrzahl der Datensätze eine Abnahme an. Unterteilt man nun dieses Sample in OECD-Länder ${ }^{7}$ und NichtOECD-Länder, so lassen sich einige weitere Punkte festhalten. So ist zu erkennen, dass in den 70er Jahren die Ungleichheit zwischen den OECD-Ländern (vgl. Abbildung 12 auf S. 56) abgenommen hat, danach unterscheiden sich die Ergebnisse zumindest für den Theil-Index wiederum sehr deutlich zwischen den einzelnen Datensätzen. Besondern auffällig ist, dass die Maddison-Zeitreihe seit Mitte der 80er Jahren einen deutlich fallenden Verlauf aufweist, was stark von den anderen Kurvenverläufen abweicht. Dies liegt darin begründet, dass für diesen Datensatz keine Beobachtungen für Luxemburg und Island vorliegen. Somit ist wiederum deutlich zu erkennen, wie sensitiv die ungewichtete internationale Ungleichheit auf Ausreißer reagiert. Betrachtet man den Verlauf der Ungleichheit für die Nicht-OECD Länder (vgl. Abbildung 13 auf S. 57), ${ }^{8}$ so sind zu Beginn des Beobachtungszeitraums ebenfalls Unterschiede zwischen den Datensätzen zu erkennen, jedoch ist seit Mitte der 80er Jahre ein Anstieg der Ungleichheit zwischen diesen Ländern zu beobachten. Da auch nach der Jahrtausendwende ein leichter Anstieg der Ungleichheit zu sehen ist, kann dies als Anzeichen dafür dienen, dass einige Länder schneller zu den Industrieländern aufholen als andere. Betrachtet man die Skalierung von Abbildung $12 \mathrm{im}$ Vergleich zu Abbildung 13 fällt des Weiteren auf, dass die Ungleichheit zwischen den OECD Ländern deutlich niedriger ist als zwischen den Nicht-OECD Ländern. Zudem sind die Differenzen in der gemessenen Ungleichheit zwischen den Datensätzen bei den Nicht-OECD Ländern fast so groß wie die gesamte gemessene Ungleichheit in den OECD Ländern. Dies ist neben den Unterschieden in der Währungsumrechnung auch darauf zurückzuführen, dass zum Jahr 1970

6. Hier ist der Sprung in der Anzahl der Beobachtungen gemeint.

7. Als OECD Länder wurden die 20 Gründungsmitglieder und die in 60er und 70er Jahren beigetretenen Länder Japan, Finnland, Australien und Neuseeland berücksichtigt.

8. Hier wurden alle Länder einbezogen, für welche seit 1970 durchgängig Daten vorlagen und die nicht zu den o.g. OECD-Ländern gehören. 
die Anzahl der Länder in den Datensätzen erheblich divergiert (vgl. Abbildung 2 auf S. 4). Damit bleibt festzuhalten, dass bei der ungewichteten internationalen Ungleichheit, die Wahl des Datensatzes auch bei einem „Balanced Panel“ zu sehr großen Abweichungen führt und keine eindeutigen Schlüsse zulässig sind. Ein weiterer Nachteil dieses Ansatzes besteht eben darin, dass durch die Auswahl der Länder zu einem gewissen Zeitpunkt, viele Staaten vollständig ignoriert werden. So ist in der obigen Untersuchung kein einziges Land des ehemaligen Warschauer Pakts berücksichtigt.

\subsection{Entwicklung der gewichteten internationalen Ungleichheit}

Die Ergebnisse der ungewichteten internationalen Ungleichheit haben einerseits gezeigt, dass sie sehr anfällig gegenüber der Auswahl der einbezogenen Länder sind, andererseits stellen sie nur einen schlechten Indikator für die Wohlfahrt der Welt dar. Schließlich ist weniger der Wohlstand der einzelnen Nationen entscheidend, sondern vielmehr der der einzelnen Bürger. Bei dem bisherigen Vorgehen wurde jedoch nur der durchschnittliche Wohlstand eines Landes berücksichtigt. Daher wird im Folgenden die gewichtete internationale Ungleichheit (Konzept 2) betrachtet, bei welcher das BIP pro Kopf eines Landes mit der Größe der Bevölkerung gewichtet wird.

Die entsprechenden Berechnungen der Gini-Koeffizienten und Theil-Indizes können der Abbildung 3 entnommen werden. Hier fallen im Vergleich zu der ungewichteten internationalen Ungleichheit zwei wesentliche Unterschiede auf. Erstens ist das Niveau der Ungleichheit im Durchschnitt höher. Dies ist ein Indiz dafür, dass die armen Länder tendenziell bevölkerungsreicher sind. So gibt es insbesondere in Europa eine Vielzahl vergleichsweise kleiner reicher Länder, während in Asien und Afrika viele große arme Nationen existieren. Zweitens ist der Verlauf der einzelnen Zeitreihen deutlich glatter als bei der vorherigen Betrachtung. Dies kann damit erklärt werden, dass bei der Gewichtung mit der Bevölkerung, das Hinzukommen und Wegfallen eines relativ bevölkerungsarmen Landes einen deutlich geringeren Einfluss ausübt. Entsprechend sind in den Zeitreihen deutlich kleinere „Zacken“ zu finden. Dennoch sind einige Zeitpunkte (Anfang der 70er Jahre und Beginn der 90er Jahre), zu denen mehrere Länder neu in den Datensatz eingetreten sind, deutlich zu erkennen.

Auch wenn sich die Punktwerte der einzelnen Zeitreihen wiederum unterscheiden, können weiterhin einige gemeinsame Trends identifiziert werden. So hat die Ungleichheit in den 60er Jahren zugenommen. In den 70er Jahren haben sich die Indikatoren kaum verändert, die Ungleichheit stagniert. Lediglich die Daten der PWT deuten auf eine weitere Abnahme hin. In den 80er Jahren hingegen ist zumindest zu Beginn und Ende ein leichtes Absinken der Ungleichheit erkennbar. Von Anfang bis Mitte der 90er Jahre nehmen sowohl Gini-Koeffizient als auch Theil-Index bei allen Datensätzen ab. Vor der Jahrtausendwende steigt die Ungleichheit bei fast allen Datenquellen kurzfristig an, um im neuen Jahrhundert wieder deutlich abzunehmen. Insgesamt ist hier das Ergebnis im Gegensatz zur ungewichteten internationalen Ungleichheit, bei der die Strukturbrüche ein wesentliches Problem darstellen, eindeutig: Im Beobachtungszeitraum hat die Ungleichheit abgenommen. Auch die sich ändernde Anzahl an Beobachtungen kann eine solche Entwicklung nicht erklären, da sich sämtliche Zeitreihen ähnlich verhalten und zu erwarten ist, dass der Effekt der höheren Anzahl an Beobachtungen 
Norbert Berthold und Alexander Brunner

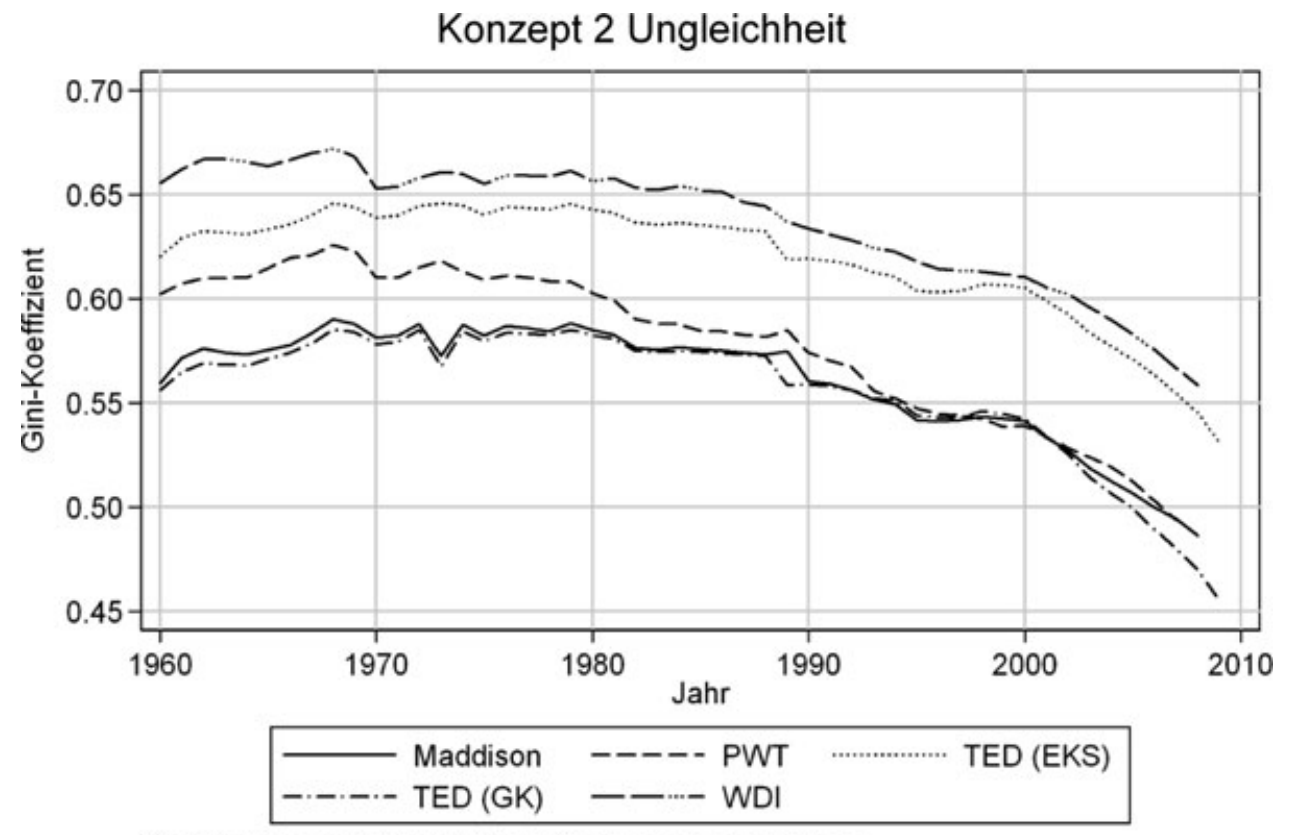

Quelle: Maddison (2010), PWT 6.3, TED, WDI; eigene Berechnungen.

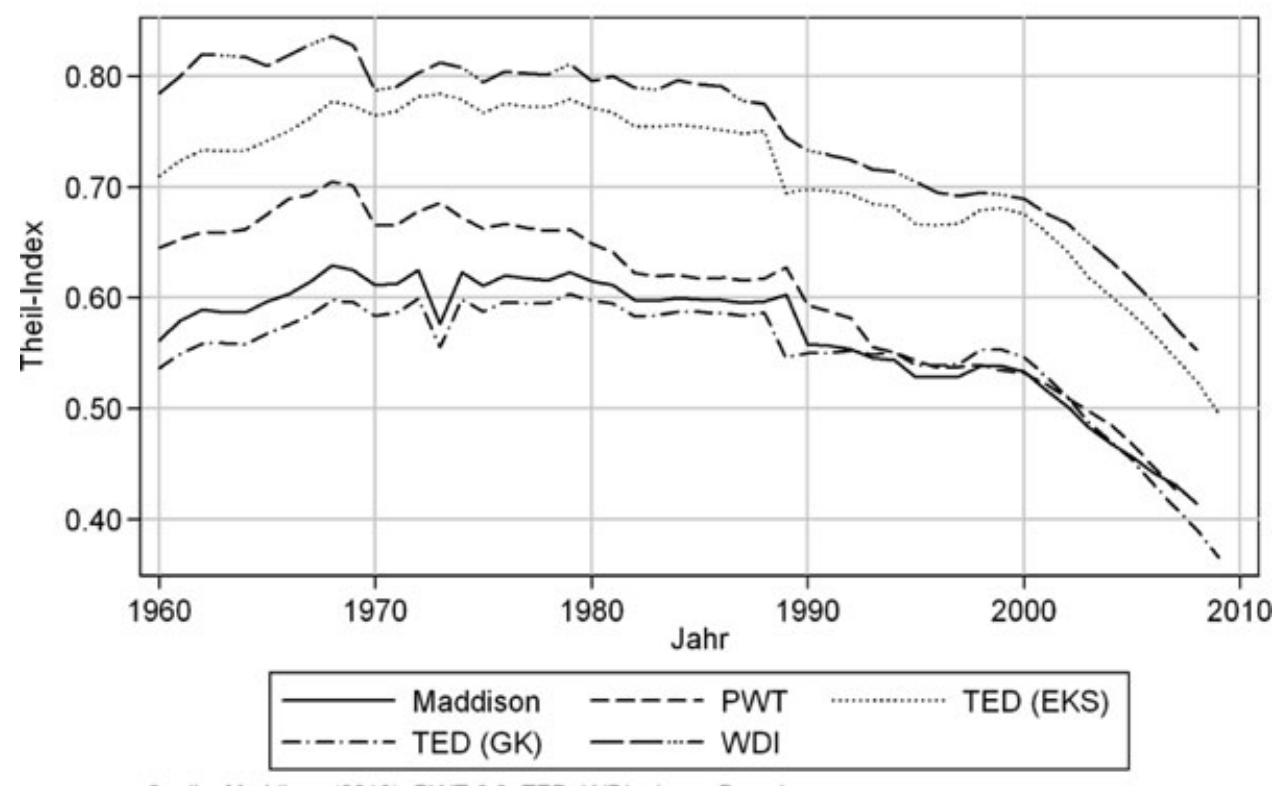

Quelle: Maddison (2010), PWT 6.3, TED, WDI; eigene Berechnungen.

Abbildung 3 Entwicklung der gewichteten internationalen Ungleichheit

eher in Richtung einer steigenden Ungleichheit geht. Lediglich die PWT-Reihe unterscheidet sich deutlich von den anderen Reihen, da hier auch in den 70er und 80er Jahren eine beständige Abnahme der Ungleichheit zu beobachten ist. 
Wie ungleich ist die Welt? Eine empirische Analyse

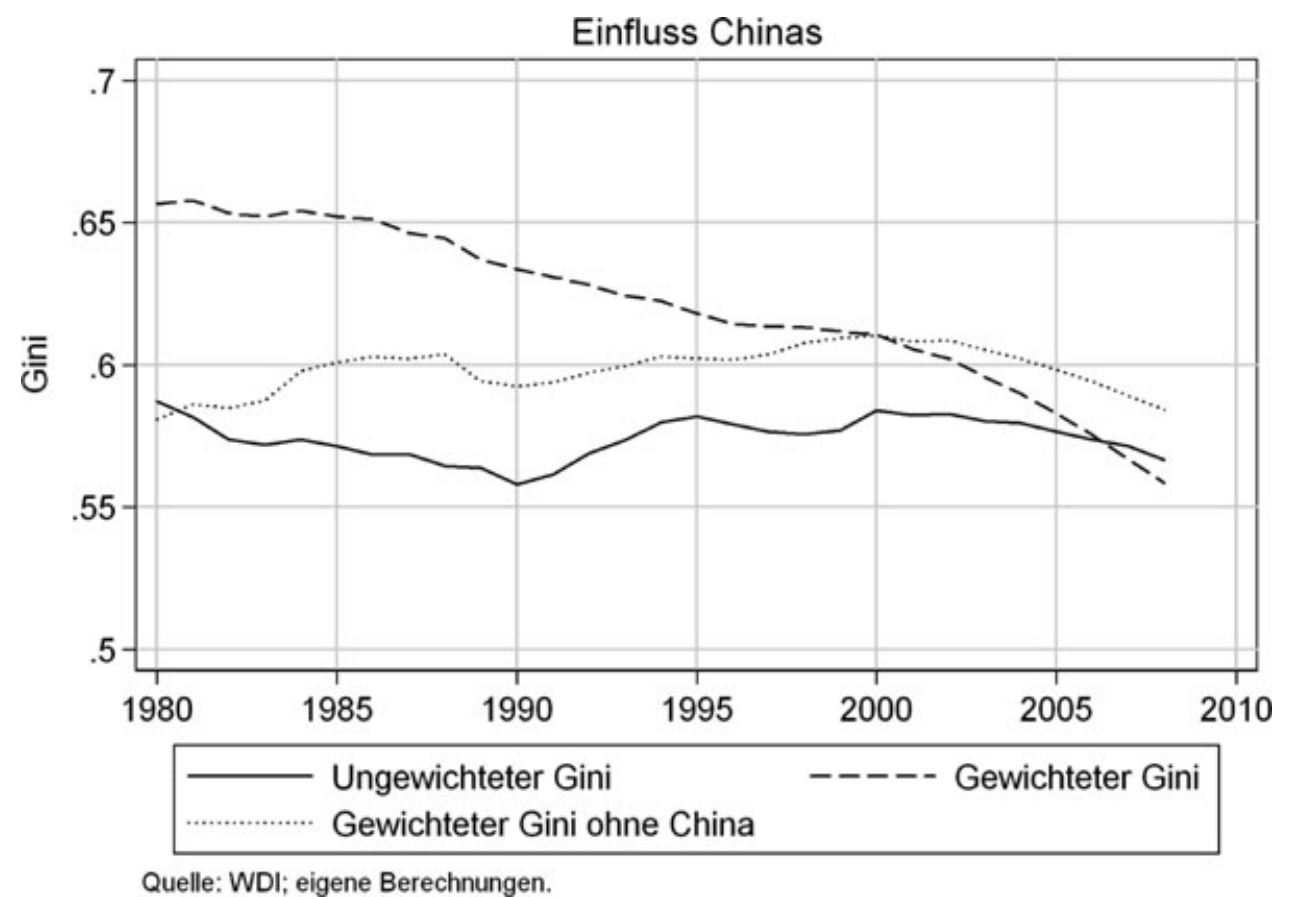

Abbildung 4 Einfluss Chinas

\subsection{Der Einfluss großer Länder auf die gewichtete internationale Ungleichheit}

Insgesamt fällt beim direkten Vergleich der gewichteten und ungewichteten internationalen Ungleichheit auf, dass diese Konzepte sich sowohl hinsichtlich des Niveaus als auch des Trends deutlich voneinander unterscheiden. Dies liegt darin begründet, dass bei der ungewichteten internationalen Ungleichheit jedes Land unabhängig von der Bevölkerung mit dem gleichen Gewicht eingeht und daher die Entwicklung von bevölkerungsreichen Ländern einen großen Einfluss hat. Hierbei fällt sofort die tragende Rolle Chinas als mögliche Erklärung auf, da es das bevölkerungsreichste Land der Welt ist und zudem in den letzten beiden Jahrzehnten deutlich an Wohlstand gewonnen hat. Diese Vermutung kann anhand der WDI Daten auch tatsächlich bestätigt werden. So ist in Abbildung 4 deutlich $\mathrm{zu}$ sehen, dass der gewichtete Gini (Konzept 2) ohne China auf einen leichten Anstieg der Ungleichheit in den 80er und 90er Jahren des letzten Jahrhunderts hindeutet. Wird China mit berücksichtigt, so ist im gleichen Zeitraum eine beständige Abnahme des Gini zu beobachten. Das neue Jahrtausend stellt dann jedoch einen Wendepunkt dar. Alle drei Zeitreihen weisen dann auf einen Rückgang der Ungleichheit hin.

Die Abnahme in der gewichteten internationalen Ungleichheit ist möglicherweise nicht nur auf China, sondern auch auf andere große Länder zurückzuführen. $\mathrm{Zu}$ nennen sind hier insbesondere Brasilien, Russland, Indien und China, die sogenannten BRIC-Staaten. Anhand der Daten der WDI kann anhand des Gini-Koeffizienten in Abbildung 5 nachvollzogen werden, wie sich die internationale Ungleichheit ohne diese Länder entwickelt hat. Hier ist zu erkennen, dass seit Beginn der 80er Jahre bis zur Mitte des neuen Jahrtausends 


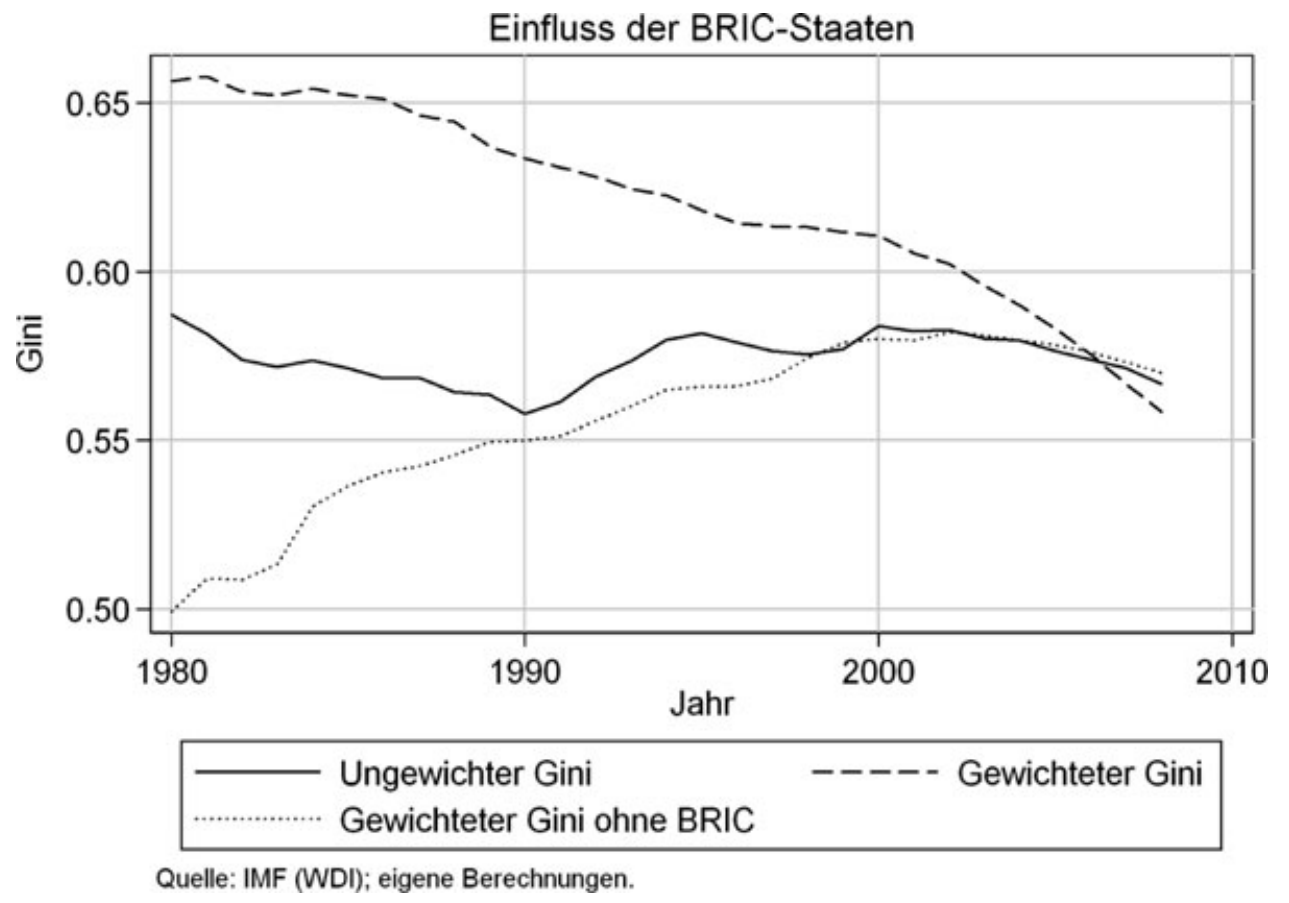

Abbildung 5 Einfluss der BRIC-Staaten

das Niveau der Ungleichheit ohne die BRIC-Staaten deutlich niedriger liegt. Auch im Trend sind Unterschiede festzustellen. Während die internationale gewichtete Ungleichheit bei Berücksichtigung dieser Länder in den 80er und 90er Jahren fällt, weist der Gini-Koeffizient ohne die BRICs auf einen Anstieg der Ungleichheit im selben Zeitraum hin. Im neuen Jahrtausend hingegen nimmt die Ungleichheit bei beiden Zeitreihen ab. Dieser Effekt ist im Datensatz mit allen Ländern jedoch schwächer ausgeprägt. Insgesamt kann somit festgehalten werden, dass auch ohne die BRIC-Staaten in den letzten Jahren die Ungleichheit deutlich abgenommen hat.

Diese Erkenntnis ist valide. Aus Abbildung 6 kann der Verlauf des gewichteten Gini-Koeffizienten und des Theil-Indexes für alle Datensätze entnommen werden. Alle zehn Zeitreihen weisen darauf hin, dass die Ungleichheit im neuen Jahrtausend abgenommen hat. Dabei unterscheidet sich das Niveau der gemessenen Ungleichheit jedoch sehrdeutlich. So beträgt der Abstand zwischen dem höchsten und dem niedrigsten beobachtbaren Wert etwa 5 Gini-Punkte. Auch im Verlauf sind Unterschiede zu erkennen. So hat die Ungleichheit bei den WDI-Daten seit Beginn der 70er Jahre stärker zugenommen als in jedem anderen Datensatz. Die Ungleichheit ist bei diesem Datensatz am aktuellen Rand am höchsten, während sie zu Beginn der 70er am unteren Rand lag. Vergleicht man Abbildung 3 mit Abbildung 6, so bleibt festzuhalten, dass die BRIC-Staaten zwar wesentlich zur Abnahme der gewichteten internationalen Ungleichheit beigetragen haben, jedoch zumindest im neuen Jahrtausend die Gleichheit auch zunahm, wenn man diese Länder vernachlässigt. Insgesamt ist somit festzuhalten, dass es zumindest im neuen Jahrtausend zu einer Abnahme der Ungleichheit kam. Dieses Ergebnis ist 
Konzept 2 Ungleichheit ohne BRIC-Staaten
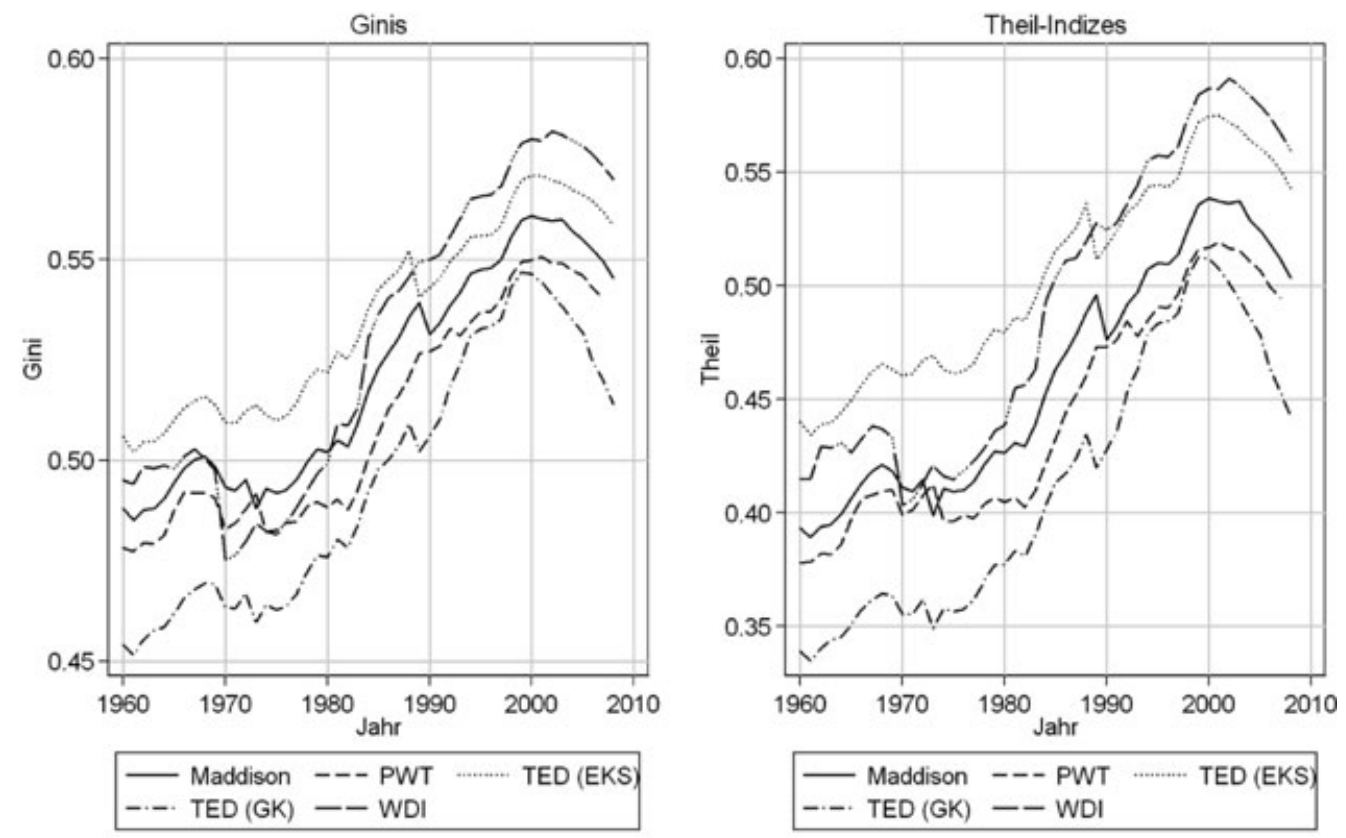

Quelle: Maddison (2010), PWT 6.3, TED, WDI; eigene Berechnungen.

Abbildung 6 Entwicklung der gewichteten internationalen Ungleichheit ohne BRIC-Staaten

für beide Definitionen der Ungleichheit, bei allen Datensätzen und selbst bei Vernachlässigung der BRIC-Staaten stabil. Das Resultat ändert sich auch dann nicht, wenn man die USA als weiteres großes Land nicht in die Berechnungen einbezieht (vgl. Abbildung 14 auf S. 57).

\subsection{Entwicklung der globalen Ungleichheit}

Im Anschluss an die Untersuchung der gewichteten und ungewichteten internationalen Ungleichheit soll nun die globale Ungleichheit (Konzept 3), also die Ungleichheit zwischen den Personen, betrachtet werden. Hierfür wird im Folgenden auf die Ergebnisse von Milanovic (2009) zurückgegriffen. Diese Ungleichheitsmaße sind dabei auf Basis der WID (World Income Distribution) ${ }^{9}$ berechnet worden. Hier liegen Daten für die Jahre 1988, 1993, 1998 und 2002 vor. Daran ist ersichtlich, dass im Vergleich zu den makroökonomischen Zeitreihen nur eine geringere Menge an Beobachtungszeitpunkten vorliegt. Für diesen Zeitraum existieren auch andere Untersuchungen, welche die personelle Ungleichheit schätzen (Bourguignon und Morrisson 2002, Sala-i-Martin 2002), jedoch steht an dieser Stelle der Vergleich mit der ungewichteten und gewichteten internationalen Ungleichheit auf Basis der Weltbankdaten im Vordergrund. Dieses Vorgehen ist deswegen konsistent, weil 
Norbert Berthold und Alexander Brunner

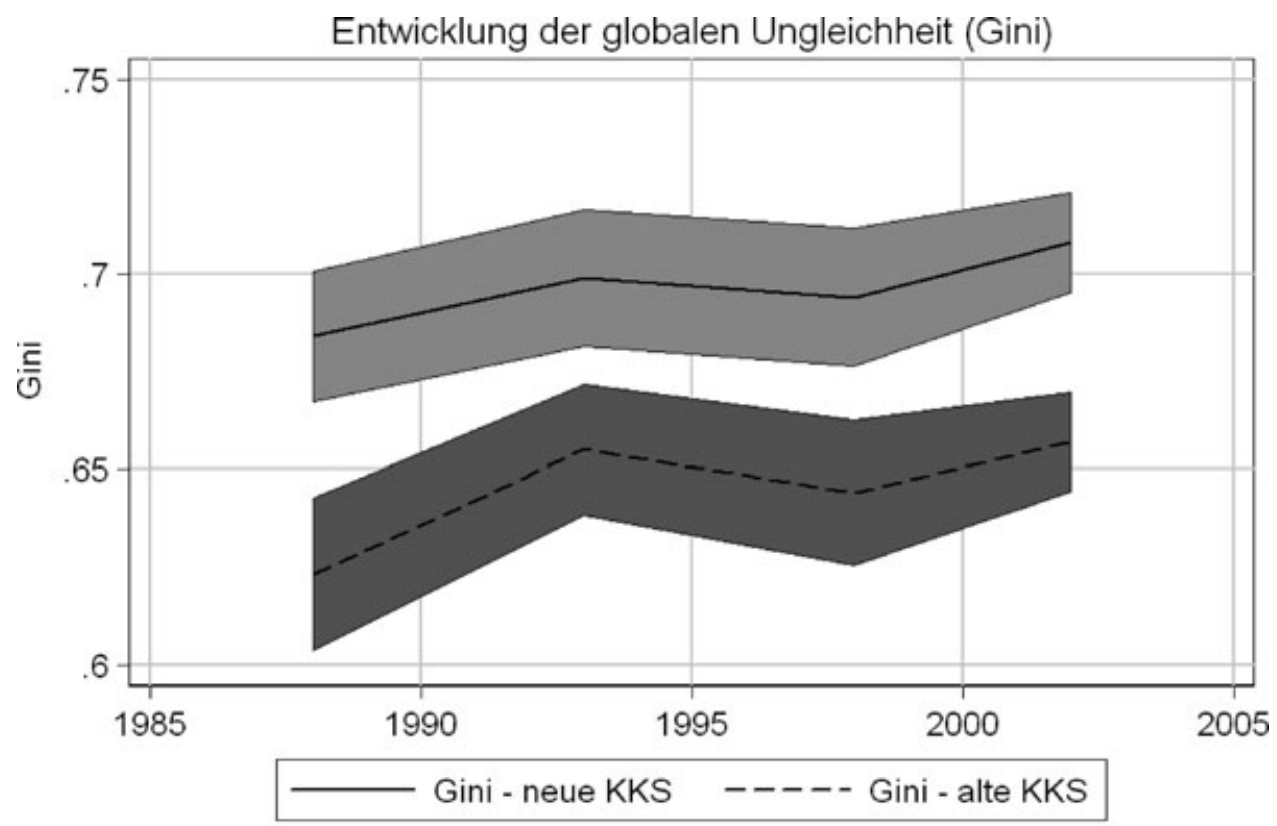

Quelle: Milanovic (2009), S. 13; eigene Darstellung.

Die Flächen geben jeweils die 1-Sigma-Konfidenzintervalle an.

Abbildung 7 Entwicklung des globalen Gini-Koeffizienten

Milanovic (2009) auch für die Berechnungen der globalen Ungleichheit die KKS der Weltbank als Grundlage nimmt.

Die von Milanovic (2009) berechneten Gini-Koeffizienten und Theil-Indizes können der Abbildung 7 und Abbildung 8 entnommen werden. Wie bereits beiden vorherigen Resultaten ist auch hier zu erkennen, dass die Unterschiede in der Berechnung der KKS einen großen Einfluss auf die gemessene Ungleichheit haben. ${ }^{10}$ Beide Indizes auf Basis der revidierten Daten der Weltbank liegen auf einem höheren Niveau als zuvor. Nichtsdestotrotz deuten alle vier Zeitreihen auf eine Zunahme der Ungleichheit zwischen 1988 und 1993 sowie 1998 und 2002 hin. Im Zeitraum 1993 bis 1998 ergeben die Daten eine Abnahme bzw. Stagnation der Ungleichheit. Dies lässt den Schluss zu, dass die personelle Ungleichheit vom Ende der 80er Jahre bis zum Anfang des neuen Jahrtausends zugenommen hat. Wenn auch die Revision der KKS zu einer Änderung des Niveaus führte, so hat sich der beobachtbare Trend nicht geändert. Es sei an dieser Stelle jedoch darauf hingewiesen, dass Sala-i-Martin (2002) bspw. zwischen Ende der 80er und Mitte der 90er Jahre eine Abnahme der Ungleichheit anhand des Gini-Koeffizienten findet. Die Ergebnisse von Bourguignon und Morrisson (2002) stimmen hingegen mit denen von Milanovic (2009) überein, da diese Autoren ebenfalls eine Zunahme des Theil-Indexes beobachten.

10. Eine Berechnung der gewichteten und ungewichteten internationalen Ungleichheit auf Basis der alten KKS war leider nicht möglich, da diese Daten nicht mehr bei den WDI verfügbar sind. Da jedoch bei den anderen Datenquellen noch keine größere Revision der KKS vorgenommen wurde, können diese als gute Proxy für den Einfluss der KKS auf die gemessene Ungleichheit dienen. 
Wie ungleich ist die Welt? Eine empirische Analyse

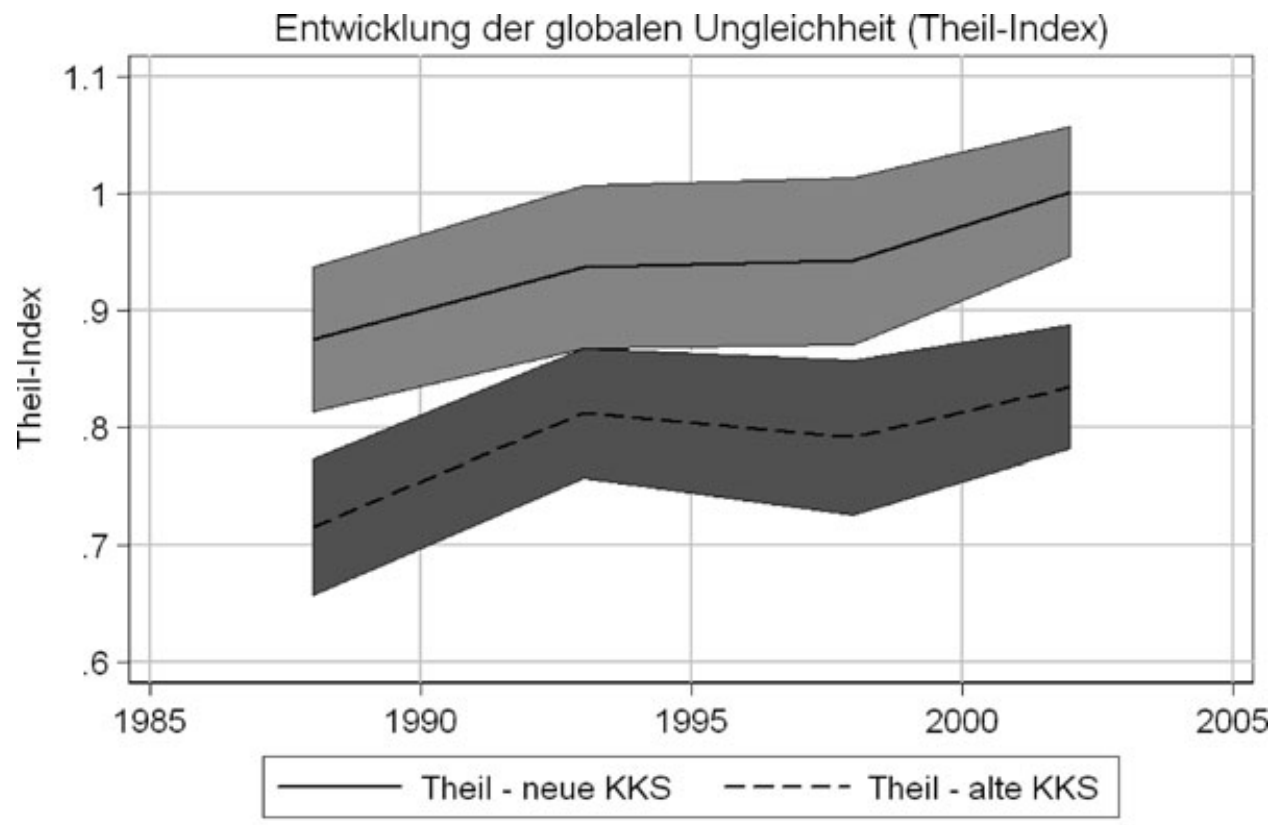

Quelle: Milanovic (2009), S. 13; eigene Darstellung.

Die Flächen geben jeweils die 1-Sigma-Konfidenzintervalle an.

Abbildung 8 Entwicklung des globalen Theil-Index

Wurden die einzelnen Konzepte bisher getrennt betrachtet und die Unterschiede bei Grundlage verschiedener Datenquellen analysiert, sollen nun die unterschiedlichen Herangehensweisen zusammengeführt werden. Entsprechend werden nun die drei unterschiedlichen Definitionen der Ungleichheit anhand der Weltbank-Daten verglichen. ${ }^{11}$ Hierzu werden die berechneten Theil-Indizes und Gini-Koeffizienten gegenüber gestellt. Zunächst soll der Gini-Koeffizient betrachtet werden (Abbildung 9). Obwohl der Gini-Index nicht zerlegbar ist (Cowell 1988), kann dessen Betrachtung zumindest ein erstes Anzeichen für das Verhältnis der internationalen und der globalen Ungleichheit liefern. Die globale und gewichtete internationale Ungleichheit liegen dabei jeweils deutlich über der gemessenen ungewichteten internationalen Ungleichheit. ${ }^{12}$

Berücksichtigt man nun die Bevölkerung der einzelnen Länder als Gewicht (Konzept 2), verändert sich das Bild deutlich. Hier ist zu sehen, dass die Ungleichheit im Laufe der Zeit deutlich abnimmt und am aktuellen Rand sogar fast dasselbe Niveau wie die ungewichtete internationale Ungleichheit erreicht hat. Diese Entwicklung ist dabei nicht nur - wie im letzten Abschnitt gezeigt wurde - dem rasanten Aufstieg Chinas und Indiens geschuldet. Da bei der internationalen Ungleichheit ein gleichverteiltes Einkommen in den Ländern unterstellt wird, unterschätzt diese die tatsächliche personelle Ungleichheit. Entsprechend ist

11. Die WDI-Daten sind hierfür geeignet, weil bei den Berechnungen der Kennzahlen der globalen Ungleichheit nach Milanovic (2009) ebenfalls auf die KKS der Weltbank zurückgegriffen wurde. Auch wenn im vorherigen Abschnitt die Probleme mit den unterschiedlichen KKS dargelegt wurden, so ist dadurch, dass die identischen KKS zugrunde liegen, zumindest eine innere Konsistenz gewährleistet.

12. Dies liegt daran, dass jedes Land mit der Gewichtung „1“ berücksichtigt wird. 


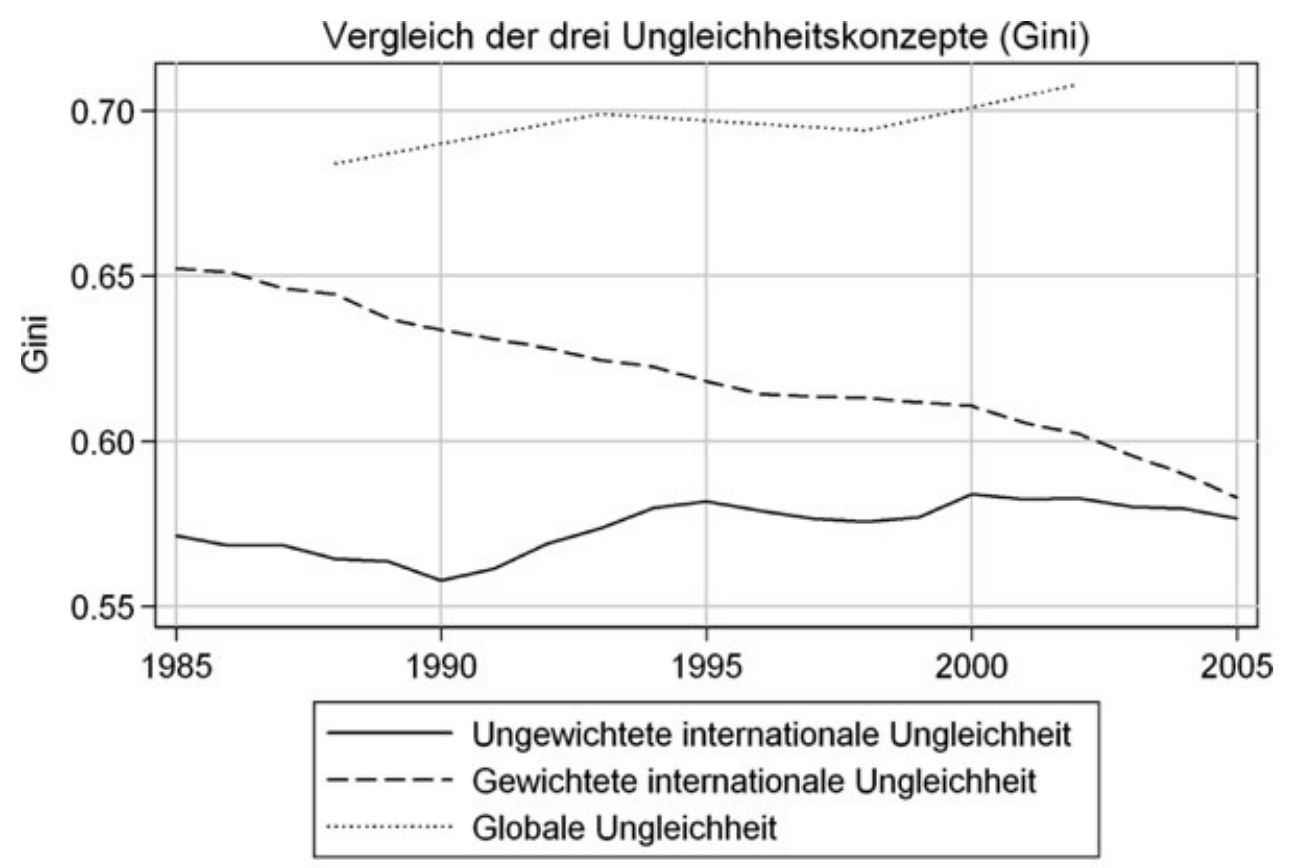

Quelle: WDI und Milanovic (2009); eigene Darstellung

Abbildung 9 Vergleich der Gini-Koeffizienten

zu erkennen, dass die globale Ungleichheit deutlich über der gewichteten internationalen Ungleichheit liegt. Zudem hat sich auch der Trend verändert. So ist zu erkennen, dass zumindest in den 15 Jahren, in denen Beobachtungen vorliegen, die Ungleichheit zugenommen hat. Dies ist nicht verwunderlich, da beispielsweise China als Land insgesamt (im Sinne des Wohlstandes pro Kopf) aufgeholt hat, dies jedoch wahrscheinlich mit einer massiven Zunahme der Ungleichheit innerhalb des Landes erkauft wurde.

Diese Feststellung wird auch bei der Analyse anhand des Theil-Indexes bestätigt. Wie bereits beim Vergleich der Gini-Koeffizienten ist zu erkennen, dass die ungewichtete internationale Ungleichheit erst leicht abnimmt, ab Anfang der 90er Jahre ansteigt und am aktuellen Rand wieder zurückgeht. Hingegen sinkt die gewichtete internationale Ungleichheit über den gesamten Zeitraum deutlich. Wiederum ist zu sehen, dass die globale Ungleichheit zwischen 1988 und 2002 ansteigt. Dieses Ergebnis steht im Widerspruch zu Bourguignon und Morrisson (2002), da diese als zentrales Ergebnis ihres Papiers feststellen, dass der Anstieg der globalen Ungleichheit insbesondere durch den Anstieg der Unterschiede zwischen den Ländern begründet liegt, während hier zwischen 1988 und 2002 ein deutlich stärkerer Anstieg bei der globalen Ungleichheit, als bei der internationalen Ungleichheit, zu erkennen ist. ${ }^{13}$ Da Bourguignon und Morrisson (2002) eine

13. Während die globale Ungleichheit zwischen 1988 und 2002 um 14,4\% anstieg, verzeichnete die ungewichtete internationale Ungleichheit nur eine Zunahme um 7,4\%. Entsprechend fiel das Verhältnis aus ungewichteter internationaler Ungleichheit zu globaler Ungleichheit von etwa 62\% auf ungefähr 58\%. 
Wie ungleich ist die Welt? Eine empirische Analyse

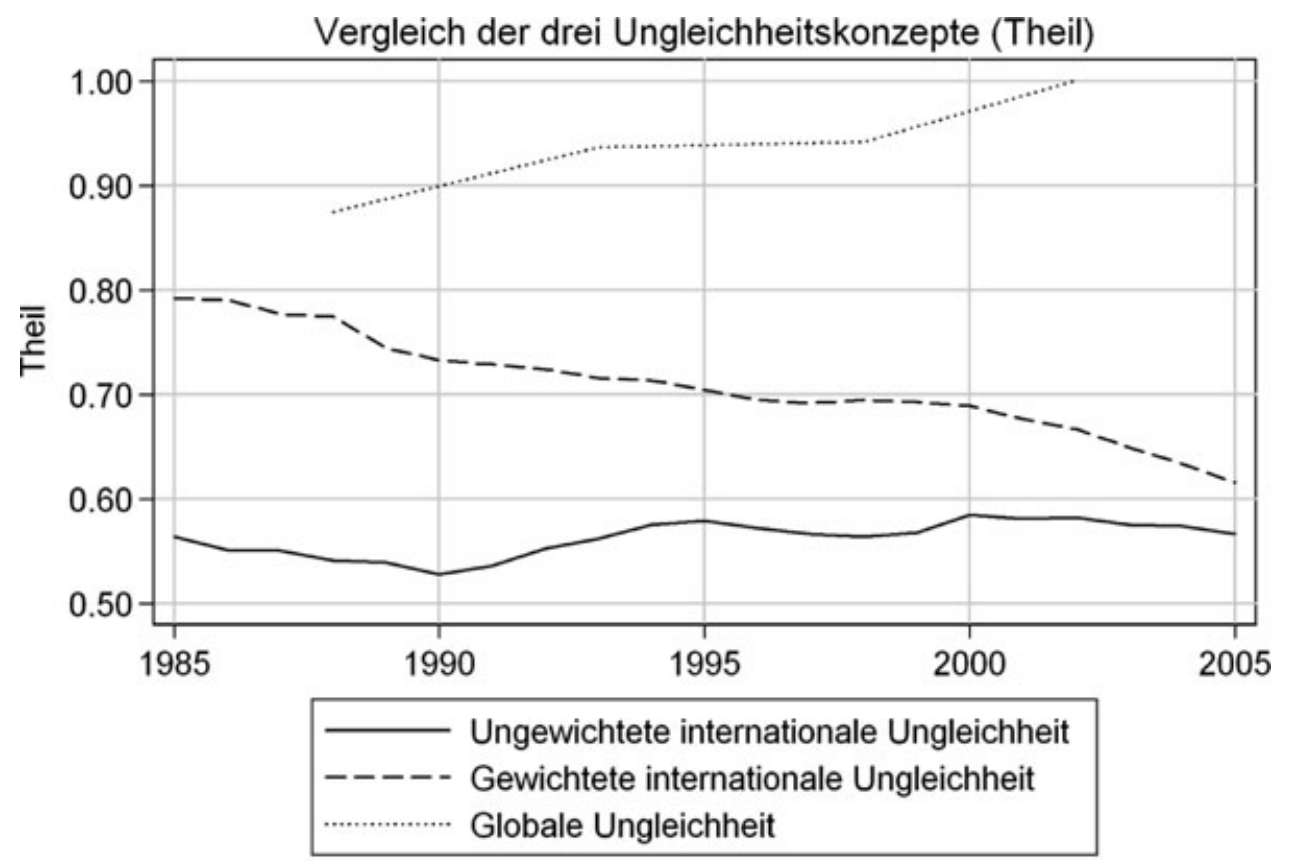

Quelle: WDI und Milanovic (2009); eigene Darstellung

Abbildung 10 Vergleich der Theil-Index

Mischung von Mikrodaten und den Makrodaten von Maddison zurückgreifen und ihre Analyse den Zeitraum 1820 bis 2002 umfasst, sind diese Abweichungen wenig verwunderlich. In Abbildung 10wird ein deutlich kürzerer Zeitraum betrachtet und es liegen zudem andere KKS zugrunde. Auch wenn an dieser Stelle keine echte Zerlegung der Ergebnisse in die Ungleichheit zwischen und innerhalb der Länder erfolgt, deuten die Ergebnisse doch darauf hin, dass die Aussage von Bourguignon und Morrisson (2002) zumindest am aktuellen Rand nicht zutreffend ist. Vielmehr kann man zum Schluss kommen, dass der Ungleichheit innerhalb der Länder eine immer größere Rolle zukommt.

Damit ist nicht bewiesen, dass die Ungleichheit innerhalb der Länder einem generellen Trend unterliegt. So finden Dollar and Kraay (2002) bei einer Untersuchung von Daten von über 100 Ländern über unterschiedlich lange Zeiträume keinen generellen Trend der Ungleichheit innerhalb der Länder. Während in einigen Ländern wie bspw. den USA und China die Ungleichheit zunahm, ist dies in anderen Nationen nicht der Fall. Andererseits betonen sowohl Milanovic (2007) als auch die OECD (2008), dass die Ungleichheit innerhalb der Länder in den letzten 20 Jahren fast überall zugenommen hat.

\section{Interpretation der Ergebnisse}

Insgesamt liefern die angestellten Untersuchungen ein diffuses Bild der Entwicklung der weltweiten Ungleichheit in den letzten Jahrzehnten. Es ist festzustellen, dass die Verwendung verschiedener Datenquellen zu abweichenden Ergebnissen führt. Insbesondere bei der ungewichteten internationalen Ungleichheit spielt die Anzahl 
der einbezogenen Länder und deren Änderung im Zeitablauf eine zentrale Rolle. Eine weitere Einflussgröße, die bei allen Konzepten der Ungleichheit auftritt, ist der Effekt der Währungsumrechnung. Die Verwendung von Kaufkraftstandards mag bei der Messung der Ungleichheit der theoretisch einzig gangbare Weg darstellen, jedoch sind in der praktischen Umsetzung die Probleme überdeutlich. Dies kann einerseits anhand der Unterschiede in den hier vorliegenden Ergebnissen abgelesen werden. Auch die Revision der Kaufkraftstandards der Weltbank zeigen, dass diese Umrechnungsfaktoren mit einer großen Unsicherheit behaftet sind (Milanovic 2009, S. 2). Dies führt dazu, dass eine Aussage über das Niveau der Ungleichheit nur schwer möglich ist. Da in diesem Papier eine Vielzahl unterschiedlicher Datenquellen genutzt werden, wird diese Schwierigkeit jedoch abgemildert, da insgesamt trotz der genannten Probleme einige gemeinsame Trends herausgearbeitet werden konnten (vgl. hierzu die Beschreibung zur Entwicklung der ungewichteten und gewichteten internationalen Ungleichheit im Kapitel 4).

Dabei kann als erstes Ergebnis festgehalten werden, dass im neuen Jahrtausend die Ungleichheit deutlich sank, während sie im vorherigen Jahrzehnt eher zugenommen hat. Dies ist insofern verwunderlich, als dass die zunehmende Globalisierung in den letzten Jahren eher als Ursache für eine Zunahme der Ungleichheit angesehen wird. Dies wird durch die vorliegenden Daten in Frage gestellt. Die zunehmende Globalisierung ging demnach nicht mit einem Anstieg, sondern einem Rückgang der Ungleichheit einher.

An zweiter Stelle tritt die Erkenntnis, dass der rasante Aufstieg Chinas und der anderen BRIC-Staaten einen sehr starken Effekt auf die Ungleichheit hat. Bei der gewichteten internationalen Ungleichheit konnte deutlich herausgestellt werden, dass die Entwicklung dieser Länder zwar einen großen Beitrag zur Zunahme der Gleichheit in den letzten fünfzehn Jahren des zwanzigsten Jahrhunderts führte. Aber auch wenn man die BRIC-Staaten und die USA, die BRIC-Staaten oder auch nur China nicht berücksichtigt, ist in den letzten zehn Jahren die Ungleichheit dennoch gesunken, was eine wesentliche Erkenntnis dieses Beitrags ist.

Drittens fällt auf, dass bei einem Vergleich der internationalen und globalen Ungleichheit zu erkennen ist, dass der Ungleichheit innerhalb der Länder eine zunehmend große Bedeutung zukommt. Es ist zumindest vorstellbar, dass Länder wie China den Aufstieg mit einer Zunahme der Ungleichheit innerhalb des Landes erkauft haben. Die Frage eines generellen Trends in der Ungleichheit der Länder ist jedoch umstritten (Dollar und Kraay 2002, Milanovic 2007, OECD 2008). Gerade deshalb, weil diese sich in den Nationen unterschiedlich entwickelt, liegt darin ein breites Forschungsfeld, das in der Zukunft vermehrt untersucht werden sollte.

Ein geflügeltes Wort sagt, dass die Sau vom Wiegen nicht fett wird. Es ist unbestritten, dass es auf der Welt weiterhin sehr arme Länder gibt und dass deren Entwicklung ein vornehmliches Interesse der Entwicklungspolitik sein muss. Die Messung der globalen Ungleichheit ist dabei jedoch nur von nachrangiger Bedeutung. Selbst wenn eine genaue Messung möglich wäre, so hätte dies keine Relevanz. Denn es gibt keine demokratisch legitimierte Weltregierung und somit auch keine Institution, die für die globale Ungleichheit in der Welt zuständig ist. Folglich ist es sinnvoll, die Ungleichheit der Weltbevölkerung auf zwei grundlegende Arten anzugehen. So ist das wichtigste Problem, die Ungleichheit zwischen den Nationen zu verstehen und zu lösen. Dies ist Aufgabe der Wachstumstheorie und deren empirischer Anwendung. Schließlich legen die 
bisherigen Überlegungen nahe, dass die globale Ungleichheit ein Konzept ist, das zwar von einem wissenschaftlichen Standpunkt aus interessant ist, jedoch in der faktischen Messung viele Schwächen offenbart. Natürlich beruht ein Großteil der globalen Ungleichheit auf den bestehenden Unterschieden zwischen den einzelnen Ländern (Bourguignon und Morrisson 2002). Jedoch hat die hier vorgenommene Untersuchung gezeigt, dass die absolute Höhe nur schwer zu erfassen ist.

Die Umrechnung der nationalen Währungen und die unterschiedliche Verfügbarkeit der Daten machen es unmöglich, ein konsistentes Bild über einen längeren Zeitraum zu zeichnen. Lediglich bei dem Trend lassen sich Gemeinsamkeiten ausmachen. Betrachtet man die Entwicklung der internationalen und globalen Ungleichheit am aktuellen Rand, so scheint dabei die Ungleichheit innerhalb der Länder eine zunehmend wichtigere Rolle zu spielen. Dabei liegt die Vermutung nahe, dass auch bei entwickelten Ländern die innere Ungleichheit zugenommen hat (OECD 2008). Gerade weil der allgemeine Trend durch einzelne Länder durchbrochen wird und viele definitorische Schwierigkeiten wegfallen, scheint die Untersuchung der Ungleichheit auf nationaler Ebene deutlich einfacher, aber auch Erfolg versprechender. Diese ermöglicht es, die Ursachen des Problems zu identifizieren und mit den Möglichkeiten des nationalen politischen Handlungsrahmens anzugehen. Die Ursachen der Ungleichheit sind so vielschichtig, dass nur eine Zerlegung dieses Problems in einen wachstumstheoretischen und national sozialpolitischen Aspekt Erfolg versprechend ist. Durch Wachstum der ärmsten Länder würde die wesentliche Ursache (Bourguignon und Morrisson 2002), nämlich die Unterschiede zwischen den Nationen, verschwinden. Bei der intranationalen Ungleichheit stellt der Arbeitsmarkt hingegen den wichtigsten Ansatzpunkt für die Bekämpfung der Ungleichheit dar. Dabei ist, entgegen der landläufigen Meinung, ein flexibler Arbeitsmarkt, in den möglichst viele Menschen integriert werden, ein probates Mittel, um Ungleichheit und Armut zu bekämpfen (OECD 2005, Burniaux, Padrini und Brandt 2006). Neben dieser statischen Perspektive spielen auch dynamische Aspekte eine Rolle: So kann eine hohe Ungleichheit in einem Jahr sozialpolitisch wenig problematisch sein, wenn gleichzeitig die Mobilität in der Gesellschaft relativ hoch ist. Jedoch deutet vieles darauf hin, dass die Länder, welche eine hohe statische Ungleichheit aufweisen, zumeist auch relativ immobil sind (Leigh 2009).

\section{Schlussbemerkungen}

Ziel dieses Papiers war es, das Ausmaß und die Entwicklung der weltweiten Ungleichheit zu untersuchen. Dies sollte insbesondere anhand der gewichteten und ungewichteten Ungleichheit mit verschiedenen Datenquellen geschehen. Die Ergebnisse sind widersprüchlich. Aufgrund unterschiedlicher Arten der Währungsumrechnung und der Anzahl der berücksichtigten Länder, schwankt die gemessene Ungleichheit stark. Jedoch konnten gemeinsame Trends identifiziert werden. Insgesamt zeichnet sich bei der ungewichteten internationalen Ungleichheit keine eindeutige Entwicklung ab. Erst wenn man den Beobachtungsraum eingrenzt, deutet einiges auf eine Zunahme der Gleichheit hin. Bei der gewichteten internationalen Ungleichheit ist eine eindeutige Abnahme der Ungleichheit über den gesamten Beobachtungszeitraum, insbesondere in den letzten 20 Jahren zu erkennen. Selbst wenn man einige Länder wie die BRIC-Staaten herausrechnet, ist zumindest noch ein deutlicher Rückgang im letzten Jahrzehnt 
festzustellen. Ein Vergleich aller drei Ungleichheitskonzepte weist schließlich darauf hin, dass ein großer Teil der Ungleichheit auf den Unterschieden zwischen den Nationen beruht, die Ungleichheit innerhalb der Länder am aktuellen Rand jedoch zunehmend an Bedeutung gewinnt.

Die Messung der globalen Ungleichheit ist weiterhin mit immensen Problemen behaftet, so dass nur in engen Grenzen Aussagen getroffen werden können. Je weiter der Blick in die Vergangenheit geht, desto unschärfer wird das gezeichnete Bild. Insgesamt liegt der Schluss nahe, das Problem der weltweiten Ungleichheit in zwei Teilbereiche zu zerlegen und diese als solche zu behandeln. Die Entwicklung der ungewichteten internationalen Ungleichheit ist dem klassischen Problem der Wachstums- und Entwicklungsökonomie zuzuordnen. Andererseits ist es viel versprechender, die unterschiedliche Entwicklung der Ungleichheit innerhalb der Länder zu untersuchen. Einerseits können dann die Gründe für die verschiedenen Trends untersucht werden, andererseits ist es dann möglich, nach Lösungen $\mathrm{zu}$ suchen, welche im Bereich der Handlungsmöglichkeiten der nationalen Sozialpolitik liegen.

Auch die Frage, wie sich die weltweite Ungleichheit in der Zukunft entwickeln wird, ist relativ unsicher. So ist das Wachstum in den Entwicklungsländern durch die Finanzkrise zunächst nicht so stark eingebrochen, wie in den entwickelten Volkswirtschaften (te Velde 2008), jedoch sind auch diese inzwischen von der dauerhaften Instabilität der entwickelten Volkswirtschaften angesteckt worden (ODI 2011). Somit besteht zwar die Chance, dass die internationale Ungleichheit in den nächsten Jahren sinkt, jedoch hängt dies sehr stark davon ab, ob die momentane Finanzkrise überwunden werden kann. Auch der Einfluss der Krise auf die Ungleichheit in den Ländern ist ungewiss (Berthold, Brunner und Zenzen 2010). Somit bleibt neben der Frage, wie ungleich die Welt nun eigentlich ist, ebenfalls im Dunkeln, wie ungleich sie sein wird.

\section{Literaturverzeichnis}

Atkinson, A.B. (1983), The Economics of Inequality. Clarendon Press, Oxford 1975, 2. Auflage 1983.

Atkinson, A.B., L. Rainwater, und T.M. Smeeding (1995), Income Distribution in OECD Countries: Evidence from the Luxembourg Income Study. OECD, Paris.

Atkinson, A.B. und A. Brandolini (2001), Promise and Pitfalls in the Use of Secondary Data Sets: Income Inequality in OECD Countries as a Case Study, Journal of Economic Literature 39, 771-799.

Berthold, N., A. Brunner und J. Zenzen (2010), Makroökonomische Rahmenbedingungen und die Einkommensverteilung: Welchen Einfluss hat die Finanzkrise?, in: T. Theurl (Hrsg.), Wirtschaftspolitische Konsequenzen der Finanz- und Wirtschaftskrise. Duncker \& Humblot, Berlin, 173-214.

Bourguignon, F. und C. Morrison (2002), Inequality Among World Citizens: 1820-1992, American Economic Review 92, 727-744.

Brümmerhoff, D. (2007), Volkswirtschaftliche Gesamtrechnungen. Oldenbourg Verlag, München 1989, 8. Auflage 2007.

Burniaux, J., F. Padrini and N. Brandt (2006), Labour Market Performance, Income Inequality and Poverty in OECD countries, OECD Economics Department Working Papers, No. 500. OECD, Paris. 


\section{Wie ungleich ist die Welt? Eine empirische Analyse}

Cowell, F.A. (1988), Inequality Decomposition: Three Bad Measures, Bulletin of Economic Research 40, 309-312.

Cowell, F.A. (2000), Measurement of Inequality, in: A. B. Atkinson und F. Bourguignon (Hrsg.), Handbook of Income Distribution. Elsevier, Amsterdam, 87-166.

Deaton, A. (2010), Price Indexes, Inequality, and the Measurement of World Poverty, Presidential Address, 17. Januar 2010, http://www.princeton.edu/deaton/downloads/ presidential\%20address\%2019january\%202010\%20all.pdf

Dollar, D. und A. Kraay (2002), Growth is Good for the Poor, Journal of Economic Growth 7, $195-225$.

Jenkins, S.P. (1991), The Measurement of Income Inequality, in: L. Osberg (Hrsg.), Economic Inequality and Poverty: International Perspectives. Sharpe, London, 3-38.

Jenkins, S.P. und P. van Kerm (2009), The Measurement of Economic Inequality, in: W. Salverda, B. Nolan und T.M. Smeeding (Hrsg.), The Oxford Handbook of Economic Inequality. Oxford University Press, Oxford, 40-67.

Leigh, A. (2009), Permanent Income Inequality: Australia, Britain, Germany, and the United States Compared, Australian National University CEPR Discussion Papers, Nr. 628, http://cbe.anu.edu.au/research/papers/ceprdpapers/DP628.pdf

Maddison, A. (2010), Statistics on World Population, GDP and Per Capita GDP, 1-2008 ad. Data. University of Groningen.

Milanovic, B. (2005), Worlds Apart: Measuring International and Global Inequality. Princeton University Press, Princeton.

Milanovic, B. (2007), Globalization and Inequality, in: D. Held and A. Kaya (Hrsg.), Global Inequality - Patterns and Explanations. Polity Press, Cambridge, 26-49.

Milanovic, B. (2009), Global Inequality Recalculated: The Effect of New 2005 PPP Estimates on Global Inequality, Working Paper 5061, World Bank. http://www.wds.worldbank.org/ external/default/WDSContentServer/WDSP/IB/2009/09/22/000158349_20090922160230 /Rendered/PDF/WPS5061.pdf

ODI (2010), The Global Financial Crisis and Developing Countries - Phase 2 Synthesis, ODI Working Papers, Nr. 316, http://www.odi.org.uk/resources/docs/5856.pdf

OECD (2005), Extending Opportunities for All - How Active Social Policy Can Benefit Us All. OECD, Paris.

OECD (2008), Growing Unequal?. OECD, Paris.

Sala-i-Martin, X. (2002), The Disturbing "Rise" of Global Income Inequality. Columbia University, New York, (Expanded Edition Published 1997).

Sen, A. (1973). On Economic Inequality. Clarendon Paperbacks, Oxford.

te Velde, D.W. (2008), The Global Financial Crisis and Developing Countries, ODI Background Note, http://www.odi.org.uk/resources/docs/3339.pdf

UN (1977). Provisional Guidelines on Statistics of the Distribution of Income, Consumption and Accumulation of Households, M. United Nations Publication, Number 61, New York.

World Bank (2006). World Development Report 2006: Equity and Development. Oxford University Press and the World Bank, Washington. 


\section{Anhang}

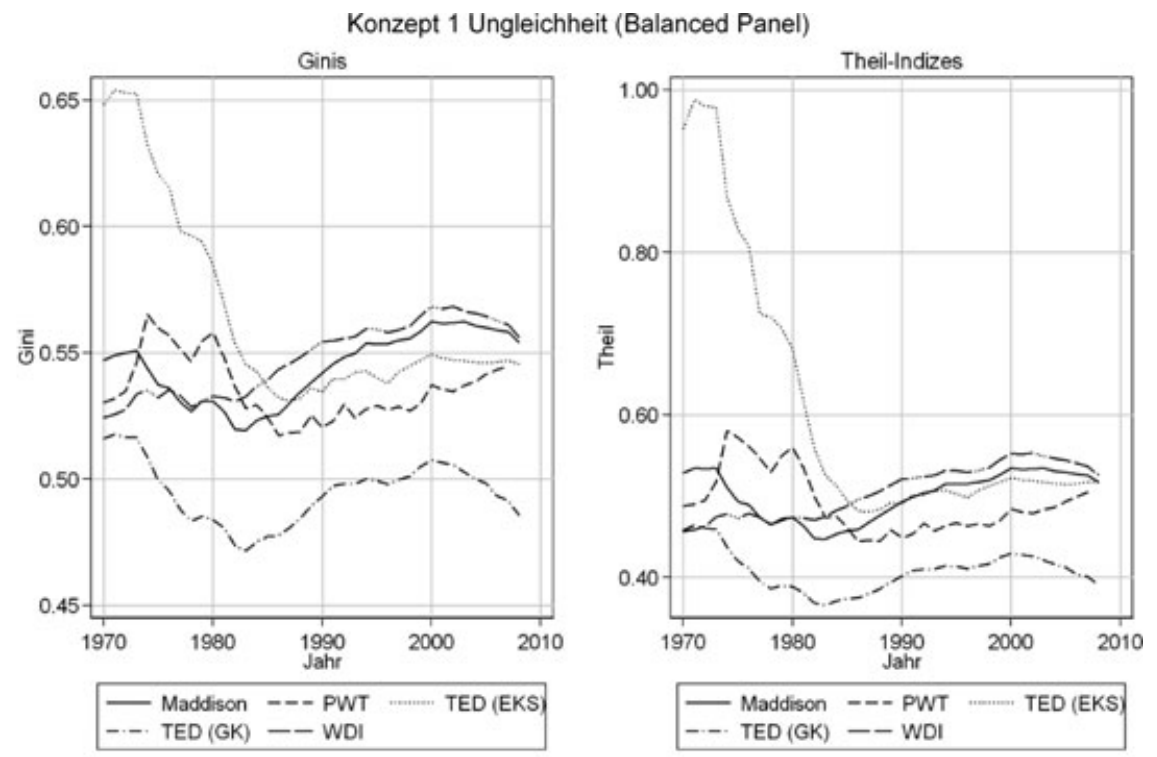

Quelle: Maddison (2010), PWT 6.3, TED, WDI; eigene Berechnungen.

Abbildung 11 Ungewichtete internationale Ungleichheit - Balanced Panel

Konzept 1 Ungleichheit (Balanced Panel - OECD-Länder)
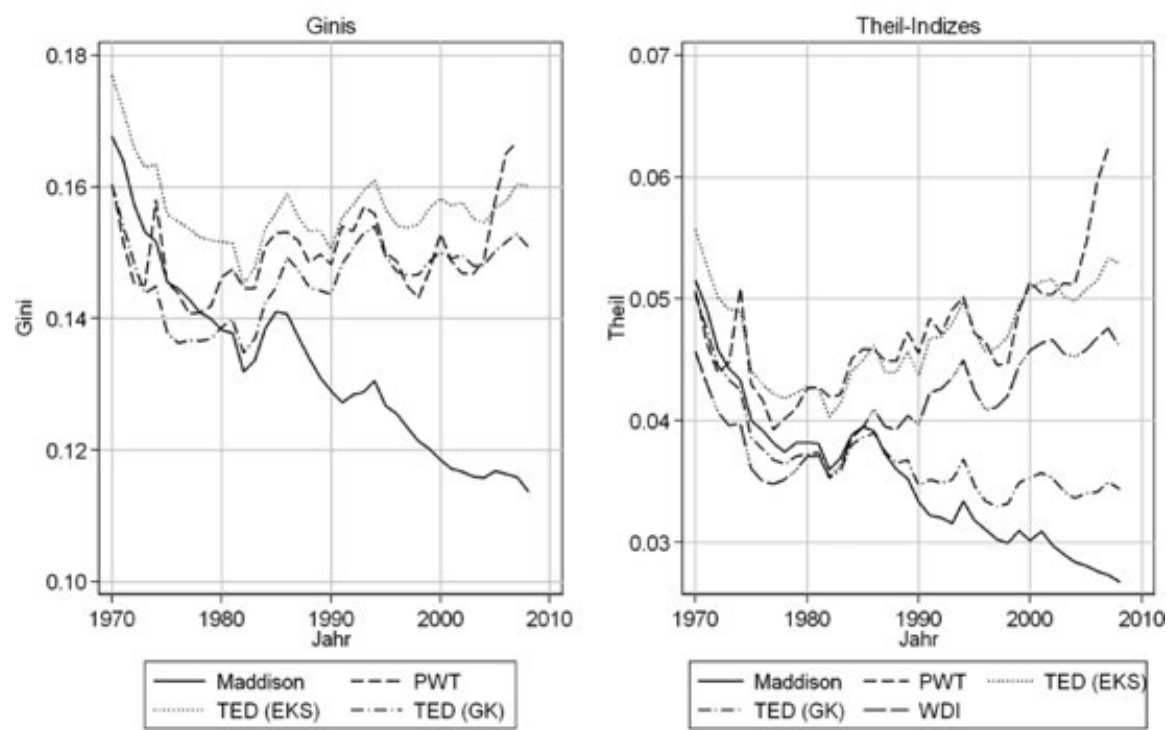

Quelle: Maddison (2010), PWT 6.3, TED, WDI; eigene Berechnungen.

Abbildung 12 Ungewichtete internationale Ungleichheit - Balanced Panel der OECD-Länder 
Wie ungleich ist die Welt? Eine empirische Analyse

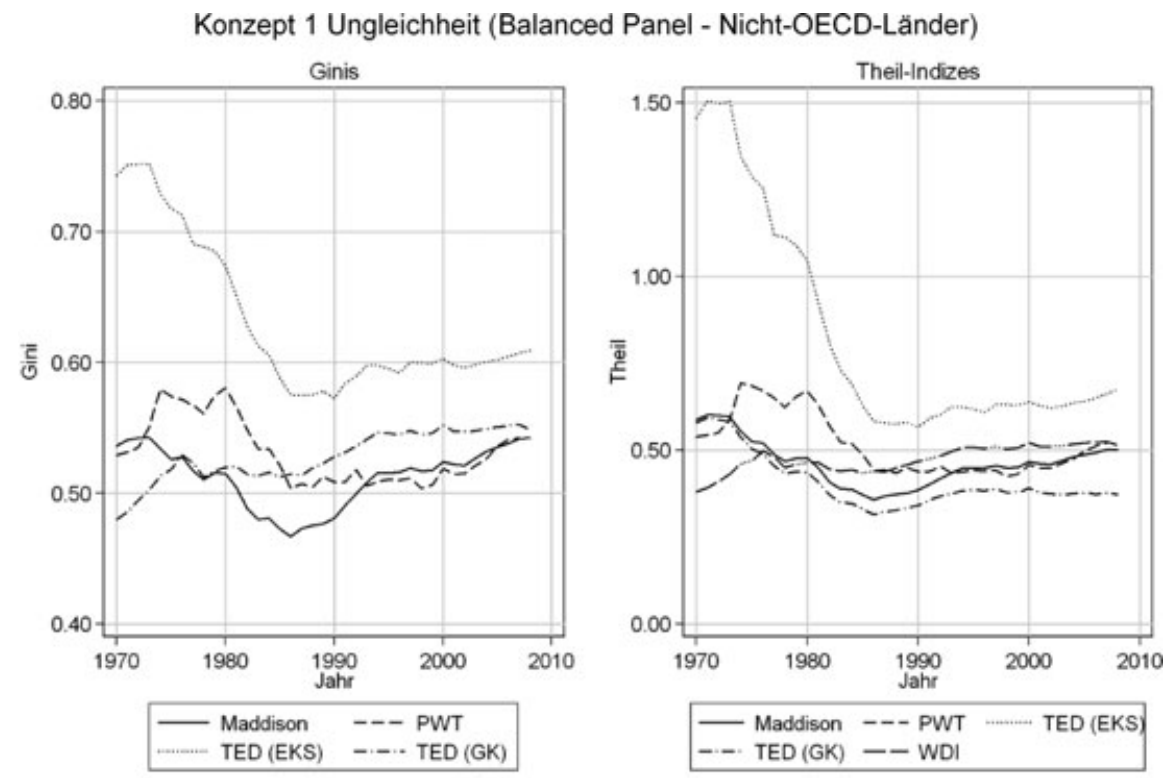

Quelle: Maddison (2010), PWT 6.3, TED, WDI; eigene Berechnungen.

Abbildung 13 Ungewichtete internationale Ungleichheit - Balanced Panel der Nicht-OECD-Länder

Konzept 2 Ungleichheit ohne BRIC-Staaten und die USA
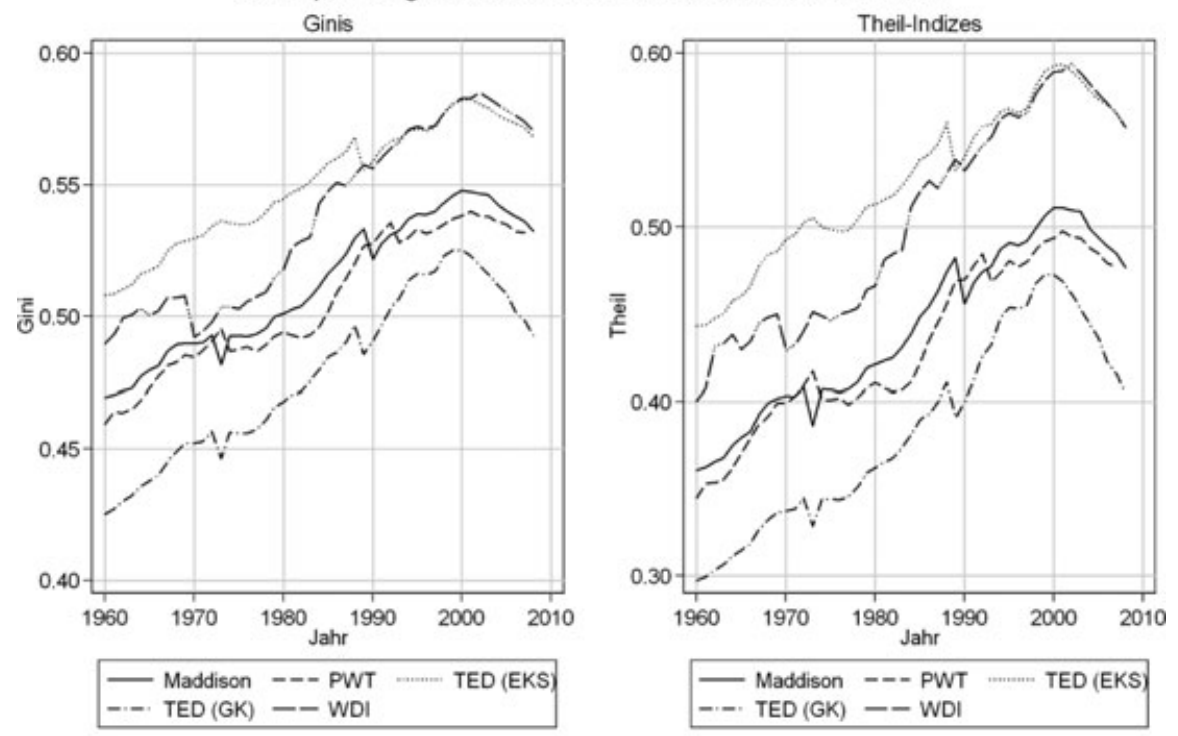

Quelle: Maddison (2010), PWT 6.3, TED, WDI; eigene Berechnungen.

Abbildung 14 Einfluss der BRIC Staaten und der USA 
Abstract: This article aims at quantifying inequality on the global level. Using different data sets we find that the results are quite contradictory. Anyhow, we can identify some common trends. Unweighted international inequality has been unambiguously falling in the last decade. In addition, weighted international inequality dropped quite dramatically in the last 30 years. The BRIC countries, especially China, were a main driver of this development. Yet excluding these countries we still find a decrease of inequality during the last decade. While inequality within countries is falling, inequality within countries seems to be on the rise and therefore will be of greater importance in future research. 